\title{
On-orbit calibration and characterization of GOES-17 ABI IR bands under dynamic thermal condition
}

\author{
Zhipeng (Ben) Wang, ${ }^{\text {a, } *}$ Xiangqian (Fred) Wu, ${ }^{\text {b }}$ Fangfang $\mathbf{Y u},{ }^{\mathrm{a}}$ \\ Jon P. Fulbright, ${ }^{\mathrm{c}}$ Elizabeth Kline, ${ }^{\mathrm{d}}$ Hyelim Yoo, ${ }^{\mathrm{a}}$ Timothy J. Schmit, ${ }^{\mathrm{e}}$ \\ Mathew M. Gunshor, ${ }^{\mathrm{f}}$ Monica Coakley, ${ }^{\mathrm{g}}$ Mason Black, ${ }^{\mathrm{g}}$ \\ Daniel T. Lindsey, ${ }^{\mathrm{h}}$ Haifeng Qian, ${ }^{\mathrm{a}} \mathrm{Xi}$ Shao,${ }^{\mathrm{a}}$ and Robbie Iacovazzi ${ }^{\mathrm{i}}$ \\ ${ }^{a}$ University of Maryland-College Park, Earth System Science Interdisciplinary Research Center, \\ College Park, Maryland, United States \\ ${ }^{\mathrm{b}}$ NOAA/NESDIS/Center for Satellite Applications and Research, College Park, Maryland, \\ United States \\ ${ }^{c}$ Arctic Slope Federal Technical Services, Seabrook, Maryland, United States \\ ${ }^{\mathrm{d}}$ NOAA/NESDIS/GOES-R Program, Greenbelt, Maryland, United States \\ ${ }^{e}$ NOAA/NESDIS/Center for Satellite Applications and Research/Advanced Satellite Products \\ Branch, Madison, Wisconsin, United States \\ ${ }^{\mathrm{f}}$ University of Wisconsin-Madison, Cooperative Institute for Meteorological Satellite Studies, \\ Madison, Wisconsin, United States \\ ${ }^{\mathrm{g}}$ Massachusetts Institute of Technology, Lincoln Laboratory, Lexington, Massachusetts, \\ United States \\ ${ }^{\mathrm{h}}$ NOAA/NESDIS/Center for Satellite Applications and Research/Regional and \\ Mesoscale Meteorology Branch, Fort Collins, Colorado, United States \\ ${ }^{\mathrm{i}}$ Global Science and Technology, Inc., Greenbelt, Maryland, United States
}

\begin{abstract}
The Advanced Baseline Imager (ABI) is a passive imaging radiometer on-board National Oceanic and Atmospheric Administration's (NOAA) Geostationary Operational Environmental Satellites-R (GOES-R) series. Its bands 7 to 16 are categorized as infrared (IR) bands, sampling within a spectral range of 3.9 to $13.3 \mu \mathrm{m}$ in mid-wave infrared (MWIR) and long-wave infrared (LWIR) regions. ABI provides variable area imagery and radiometric information of Earth's surface, atmosphere, and cloud cover. All of the IR bands are calibrated on-orbit in reference to an internal blackbody. While the ABI aboard the GOES-16 satellite has been working properly, an anomaly with GOES-17 ABI's cooling system, specifically its loop heat pipe (LHP) subsystem, prevents heat from being efficiently transferred from the ABI electronics to the radiator to be dissipated into space. As a consequence, the heat accumulates inside the instrument, so the temperatures of its key components for IR calibration, including the focal plane modules (FPMs), scan mirrors, and blackbody, cannot be maintained at their designed operational levels. As an example, the temperatures of MWIR and LWIR FPMs, where IR detectors are located, are currently operated at a baseline temperature of $\sim 20 \mathrm{~K}$ warmer than the design and vary by as many as $27 \mathrm{~K}$ diurnally. This causes severe degradation to the data quality of ABI IR Level $1 \mathrm{~b}$ radiance and subsequent Level 2+ products during the hot period of the day. Significant progress has been made to mitigate the effects of the LHP anomaly to optimize the IR performance of GOES-17 ABI. We summarize the efforts made by NOAA's GOES-R Calibration Working Group, working collaboratively with other teams, to evaluate and alleviate the negative impacts of warmer and floating FPM temperatures on ABI IR calibration, and assess the IR performance accordingly. (C) The Authors. Published by SPIE under a Creative Commons Attribution 4.0 Unported License. Distribution or reproduction of this work in whole or in part requires full attribution of the original publication, including its DOI. [DOI: 10.1117/1.JRS.14.034527]
\end{abstract}

Keywords: Geostationary Operational Environmental Satellites-R; Advanced Baseline Imager; sensor; radiometric calibration; infrared.

Paper 200365 received May 12, 2020; accepted for publication Jul. 31, 2020; published online Sep. 30, 2020.

\footnotetext{
*Address all correspondence to Zhipeng (Ben) Wang, E-mail: zhipeng.wang@noaa.gov

(C) 2020 Massachusetts Institute of Technology.
} 


\section{Introduction}

The Advanced Baseline Imager (ABI) is the main payload aboard National Oceanic and Atmospheric Administration's (NOAA) Geostationary Operational Environmental Satellites$\mathrm{R}$ (GOES-R) series. It remotely collects data in geosynchronous orbit of the land, ocean surface, and atmosphere of the Earth's Western Hemisphere in higher radiometric sensitivity and spectral/spatial/temporal resolutions compared with the legacy GOES imager, for weather forecasting and other Earth science applications. ${ }^{1,2}$ The first satellite in the series, GOES-R, was launched in November 2016 and renamed GOES-16 upon reaching geostationary orbit. In December 2017, it was declared fully operational as NOAA's GOES-East satellite. The second in the series, GOES-S, was launched in March 2018, later renamed GOES-17, and declared operational as GOES-West in February 2019. Located at $75.2 \mathrm{deg} \mathrm{W}$ and $137.2 \mathrm{deg} \mathrm{W}$, respectively, the two satellites can continuously monitor the United States and its neighboring regions. The next two satellites of the series, GOES-T and GOES-U, are currently scheduled to launch in 2021 and 2024, respectively.

ABI, manufactured by the L3Harris Corporation, is a multiband, two-axis-scanning passive imaging radiometer. Its scanner consists of two scan mirrors that can independently move the line-of-sight (LOS) of the instrument along the East-West (EW) and North-South (NS) directions to view the Earth in three standard geographic coverage regions: full disk (FD) of Western Hemisphere, contiguous United States (CONUS), and smaller mesoscale (MESO) images of areas where storm activity or other rapidly changing phenomena are monitored. ${ }^{3}$ ABI has 16 spectral bands, compared with five on the previous generation of the GOES imager, collecting data in six visible/near-infrared (VNIR) bands 1 to 6 and 10 infrared (IR) bands 7 to 16. Some key design specifications (Spec.) and technical features of these bands, including the center wavelength, nadir spatial resolution, and number of detectors, are summarized in Table 1.,5 $\mathrm{ABI}$ is a mission-critical payload for the GOES-R series, providing more than $65 \%$ of all mission data products currently defined. ${ }^{6}$

Table $1 \mathrm{ABI}$ spectral band design.

\begin{tabular}{|c|c|c|c|c|}
\hline Band & $\begin{array}{c}\text { Wavelength } \\
(\mu \mathrm{m})\end{array}$ & $\begin{array}{l}\text { Resolution } \\
(\mathrm{km})\end{array}$ & $\begin{array}{l}\text { No. of detector } \\
\text { rows }\end{array}$ & Typical applications \\
\hline 1 & 0.47 & 1 & 676 & Aerosols \\
\hline 2 & 0.64 & 0.5 & 1460 & Clouds \\
\hline 3 & 0.86 & 1 & 676 & Vegetation \\
\hline 4 & 1.38 & 2 & 372 & Cirrus \\
\hline 5 & 1.6 & 1 & 676 & Snow/ice discrimination and cloud phase \\
\hline 6 & 2.2 & 2 & 372 & Cloud particle size, snow, and cloud phase \\
\hline 7 & 3.9 & 2 & 332 & Fog, stratus, fire, and winds \\
\hline 8 & 6.2 & 2 & 332 & High-level water vapor, rainfall, and winds \\
\hline 9 & 6.9 & 2 & 332 & Mid-level water vapor, rainfall, and winds \\
\hline 10 & 7.3 & 2 & 332 & Low-level water vapor and winds \\
\hline 11 & 8.4 & 2 & 332 & Cloud-top phase, dust, and rainfall \\
\hline 12 & 9.6 & 2 & 332 & Total column ozone and turbulence \\
\hline 13 & 10.3 & 2 & 408 & Clouds and surface \\
\hline 14 & 11.2 & 2 & 408 & Clouds and sea surface temperatures \\
\hline 15 & 12.3 & 2 & 408 & Clouds and sea surface temperatures \\
\hline 16 & 13.3 & 2 & 408 & Cloud-top pressure \\
\hline
\end{tabular}


ABI contains three focal plane modules (FPMs) that filter the incident light into its spectral bands and convert it into analog signal. The FPMs map into three spectral regions: a VNIR module consisting of bands 1 to 6 , a mid-wave infrared (MWIR) module consisting of bands 7 to 11 , and a long-wave infrared (LWIR) module consisting of bands 12 to 16 . The whole modules, including the detectors assembled therein, are cryogenically cooled for higher responsivity and reduced thermally induced noise. Both the MWIR and LWIR FPMs are nominally controlled at $\sim 60 \mathrm{~K}$ by design, and the VNIR FPM is nominally controlled at $\sim 170 \mathrm{~K}$. As this paper focuses on IR performance, "FPM" refers to the MWIR and LWIR FPMs unless stated otherwise. All ABI electronics, FPM included, are side1/side2 redundant, with side 2 in operation for both ABIs since launch.

The analog output of the ABI detectors is digitized by a 14-bit analog-to-digital (A/D) converter before being compressed, packetized, and downlinked to the ground system (GS) as Level 0 (L0) raw data. The GS runs ground processing algorithms (GPAs) to convert L0 data into Level 1b (L1b) radiance data by applying radiometric and geometric correction and then into Level 2+ (L2+) products. Radiometric calibration is a component of the L1b GPA, aiming to scale the digital response of each detector element into spectral radiance and subsequently for IR bands, brightness temperature (BT). ABI has on-orbit calibration capability for all of its 16 bands. The calibration methodology is similar to other remote sensing spectroradiometers, such as the Moderate Resolution Imaging Spectroradiometer (MODIS) instruments aboard NASA's Terra and Aqua satellites $^{7}$ and the Visible Infrared Imaging Radiometer Suite (VIIRS) instruments aboard NOAA's Suomi-NPP and NOAA-20 satellites. ${ }^{8,9}$ For IR bands, the calibration primarily references an on-board temperature-controlled blackbody that provides a radiance standard and deep space that provides the detector dark offset. The linear gain coefficient is automatically updated every blackbody observation while the nonlinear coefficients are static. On-orbit calibration is essential to maintaining the L1b quality of ABI IR bands to compensate for any on-orbit drift of the instrument performance. Comprehensive Post Launch Testing (PLT) has been conducted to validate or update the calibration parameters determined prelaunch to establish a baseline for on-orbit calibration. This effort was led by the GOES-R Flight Project and L3Harris. ${ }^{10}$

During the PLT stage of GOES-17 ABI, it was discovered that its loop heat pipe (LHP), a subsystem of the cooling system that transfers heat from the ABI electronics to the radiator, was not operating at its full capacity. Therefore, during local nighttime, the Sun may heat up the instrument faster than it can be cooled and the temperatures of the FPMs cannot be maintained at their designated levels. This has led to partial loss of ABI IR imagery at wavelengths longer than $4 \mu \mathrm{m}$ and serious degradation of the quality of the remainder, the degrees of which are based on the orbital conditions of solar illumination that oscillate both diurnally and seasonally. Significant progress has been made to increase the data availability and improve the image quality through the optimization of the instrument settings and the revision of ground processing algorithm. ${ }^{11-13}$ This paper summarizes the efforts made by the GOES-R Calibration Working Group (CWG), who leads the ABI Post Launch Product Tests (PLPTs) related to L1b products, contributes to the monitoring and improvement of $\mathrm{ABI}$ calibration, and studies the relationship between the IR performance of GOES-17 ABI and the FPM temperature to mitigate its negative impacts. ${ }^{14-18}$ This paper starts with an introduction of ABI IR calibration methodology and the LHP anomaly in Sec. 2. The radiometric bias introduced by the fluctuation of the FPM temperature is described in Sec. 3, together with its mitigation by the predictive calibration (pCal) algorithm developed by L3Harris and the GOES-R Program. The relationship between the key metrics used to characterize IR detector performance, such as noise and dynamic range, and FPM temperature are detailed in Sec. 4. An overview of IR performance of GOES-17 ABI is also provided and is followed by the summary in Sec. 5 .

\section{ABI IR Calibration Overview and GOES-17 ABI LHP Anomaly}

\section{1 $A B I I R$ Calibration Strategy}

Similar to other IR sensors, ABI uses a quadratic equation to relate the output of a detector in digital count to the incident spectral radiance. The ABI calibration algorithm is developed to calibrate the radiometric response of each detector element by computing the linear detector 
gain coefficient $m$ and then using the coefficient to compute the spectral radiance from the detector's Earth view (EV) samples. The approach of two-point calibration is adopted; this consists of measuring the responses of detector elements to the observations of a temperature-controlled blackbody, referred to as internal calibration target (ICT), and deep space, referred to as spacelook (SL). The thermal emission from deep space is essentially zero. The difference between the detector's responses to the ICT look and the SL then reflects the incident radiance from the ICT, $L_{\mathrm{ICT}}^{\text {eff }}$, subject to the adjustment of the thermal contribution from the scan mirrors. The ICT temperature is measured by 12 embedded platinum resistance thermometers with high accuracy in nearly real time. With ICT temperature known, the thermal emission from the ICT can be accurately determined through Planck's equation. The contribution to the incident radiance from the scan mirrors is also computable since the temperatures of both scan mirrors are measured by affixed thermistors and the scan-angle-dependent emissivity of the scan mirrors was characterized prelaunch. With all of these compensations taken into account, $m$ is regularly updated onorbit for each band and detector as

$$
m=\frac{L_{\mathrm{ICT}}^{\mathrm{eff}}+\left(L_{\mathrm{EW} \text { at ICT }}^{\mathrm{eff}}+L_{\mathrm{NS} \text { at ICT }}^{\mathrm{eff}}\right)-\left(L_{\mathrm{EW} \mathrm{at} \mathrm{Space}}^{\mathrm{eff}}+L_{\mathrm{NS} \text { at Space }}^{\mathrm{eff}}\right)-Q \cdot\left(\bar{x}_{\mathrm{ICT}}-\bar{x}_{\text {space }}\right)^{2}}{\bar{x}_{\mathrm{ICT}}-\bar{x}_{\text {space }}},
$$

where the quadratic coefficient $Q$ is measured from prelaunch characterization and supplied in a lookup table (LUT) for on-orbit calibration. $Q$ is not regularly updated on-orbit mainly because the contribution of the nonlinear term to the total radiance is much smaller compared with the linear term. The $\bar{x}$ is the average detector counts over multiple samples. $L_{\mathrm{NS}}^{\text {eff }}$ and $L_{\mathrm{EW}}^{\text {eff }}$ are the effective self-emission spectral radiance for the NS and EW scan mirrors, respectively. Their subscripts of ICT or space indicate that the measurements of the variables are for ICT look or SL. The update of $m$ is conducted for each ICT observation, currently set at every $10 \mathrm{~min}$ for GOES-16 and 5 min for GOES-17. It is worth noting that while $m$ is conventionally named the gain coefficient, it actually carries units of the inverse of the detector gain.

With the calibrated $m$, the radiance of each EV sample is computed by

$L_{\mathrm{EV}}$

$=\frac{m \cdot\left(x_{\text {sample }}-\bar{x}_{\text {space }}\right)+Q \cdot\left(x_{\text {sample }}-\bar{x}_{\text {space }}\right)^{2}-\left(L_{\mathrm{EWatsample}}^{\mathrm{eff}}-L_{\mathrm{EW} \text { atspace }}^{\mathrm{eff}}\right)-\left(L_{\mathrm{NS} \text { atsample }}^{\mathrm{eff}}-L_{\mathrm{NS} \text { atspace }}^{\mathrm{eff}}\right)}{\rho_{\mathrm{EWatsample}} \cdot \rho_{\mathrm{NS} \text { at sample }}}$,

where $x_{\text {sample }}$ is the detector count of EV samples and $\rho_{\mathrm{NS} \text { at sample }}$ and $\rho_{\mathrm{EW}}$ at sample are the reflectivity, calculated as one minus the emissivity, of the scan mirrors at the scan angles of EV samples. For IR bands, the radiance is then converted into BT by Planck's equation with the band-average spectral response function (SRF) characterized prelaunch as input.

The ABI IR calibration is designed to be performed in a relatively stable thermal environment: the ICT temperature is nominally controlled at $302 \mathrm{~K}$ and the FPM temperature is nominally controlled at $60 \mathrm{~K}$. The ICT temperature control is achieved with an internally embedded heater plus the temperature feedback circuit. However, it is noteworthy that such temperature control is not requisite for the calibration in Eqs. (1) and (2) to hold. Only if the ICT temperature and other input variables in the equations are accurately and timely measured will the IR calibration remain valid.

$\mathrm{ABI}$ on-orbit calibration is part of the L1b production process conducted at the GS automatically. The calibration-related variables $x_{\mathrm{ICT}}, x_{\mathrm{SL}}, m$, and miscellaneous telemetry temperatures are output by the GS as calibration data files INST-CAL and INST-CAL-ENG, enabling fast and in-depth assessment of calibration performance and anomaly investigation. Intercomparison between the L1b radiances of GOES-17 and GOES-16 ABIs, or between the ABI and other well-calibrated IR sensors, such as the infrared atmospheric sounding interferometer (IASI) or the Cross-track Infrared Sounder (CrIS), can determine the calibration accuracy and detect anomalies in a more straightforward fashion. These two offline analytical strategies are the cornerstones for the evaluation and improvement of ABI IR calibration by the CWG. 


\subsection{GOES-17 LHP Malfunction and Resulting Anomalous FPM Temperatures}

ABI IR detectors are cryogenic detectors, offering critical advantages of high responsivity and low noise over noncryogenic ones. ${ }^{11}$ The ABI FPM temperatures are designed to be controlled at cryogenically low temperatures with tight stability to maintain radiometric accuracy. The thermal control of the FPMs is accomplished by an active cryocooler subsystem. Excess thermal energy ejected by the cooler, mostly from direct solar illumination, needs to be transferred to a radiator to be dumped into space. The interface between the cooler and the radiator is the LHP, inside which working coolant is vaporized at one end and condensed at the other to transport heat away. By procedure, the LHP is enabled by operations after the outgas phase of the mission is complete and should remain on when the ABI is operating normally. As its other key electronics components, ABI has two redundant cryocoolers and LHPs, which can be operated individually or together but are controlled by either electronic side.

Soon after the LHP was commanded to start for GOES-17 ABI, it was realized that the instrument was not cooled properly. Subsequent on-orbit troubleshooting and investigation revealed the root cause to be a blockage of the LHP that prevents the circulation of coolant from running at its full capacity. As a consequence, the substantial heat load from the Sun overwhelms the limited heat-carrying capacity of the anomalous LHP, hindering adequate cooling for the instrument. The FPM temperature cannot stabilize at the designated setting temperature of $60 \mathrm{~K}$ for a full 24-h period because the cryocooler power must be ramped down during times of increased solar load to prevent it from reaching its critical operating temperature. This in turn allows the FPM temperature to begin to increase. In addition to potential damages to the hardware by overheating, the IR detector performance can drop substantially at higher temperatures, even to the level of a complete loss of EV images. Several operational strategies have been employed to relieve the heat buildup and alleviate its damage to the instrument: a satellite yaw flip is executed biannually to minimize direct solar illumination into the instrument; both primary and redundant cryocoolers/LHPs are running simultaneously to maximize the heat outflow; the telemetry alarm limits are increased, so the cryocoolers can run full time; the baseline operating temperature of the MWIR/LWIR FPMs is raised to $81 \mathrm{~K}$. The performance improvement through these operations had been so remarkable that the instrument could be declared as operational as GOES-West with the vast majority of data availability restored. ${ }^{19}$

Even with all of the progress made, the FPM temperatures of GOES-17 ABI still deviate from its $81 \mathrm{~K}$ baseline and fluctuate with solar illumination conditions.

1. Figure 1 shows the typical diurnal variation of the FPM temperatures and other key telemetry temperatures related to IR calibration. On each day during the local nighttime hours, the Sun moves across the field of regard (FOR) of ABI from the opposite side of the Earth, as shown in Fig. 2. The solar energy enters the optical aperture of the instrument, creating more heat than the LHP can transport away. The cryocoolers are forced to be increased in power to maintain the $81 \mathrm{~K}$ FPM temperature, until reaching their maximum operating temperature, at which time their power is reduced, allowing the FPM temperatures to begin to rise. This typically occurs after local midnight (0900 UTC) but does vary seasonally. The heat load then builds up at a faster speed at the FPM and warms it up, starting from $\sim 1000$ UTC. The maximum for the day of $98 \mathrm{~K}$ is reached at around 1300 UTC before the temperature drifts lower. During the local daytime centered at 2100 UTC, the Sun is at the back of ABI. Direct solar illumination is minimized, so the FPM temperatures can stabilize to the $81 \mathrm{~K}$ baseline. The temperatures of ICT and scan mirrors also exhibit diurnal variation patterns. The temperature patterns shown in the plots are typical and repeat from day to day. However, the magnitudes and the duration of the temperature drift oscillate seasonally.

2. Every year, the vertical positions of the Sun moves up and down (Fig. 2). The so-called eclipse seasons centering at spring and fall Equinox refer to the period when the Sun moves into the blind spots of ABI for part of the day. The reception of the sunlight, or the FPM temperature as a result, reaches its maximum at the start and end of the eclipse seasons. When the sunlight is partially blocked by the Earth during the eclipse season or the Sun is far away from the FOR of the ABI, the solar illumination into 

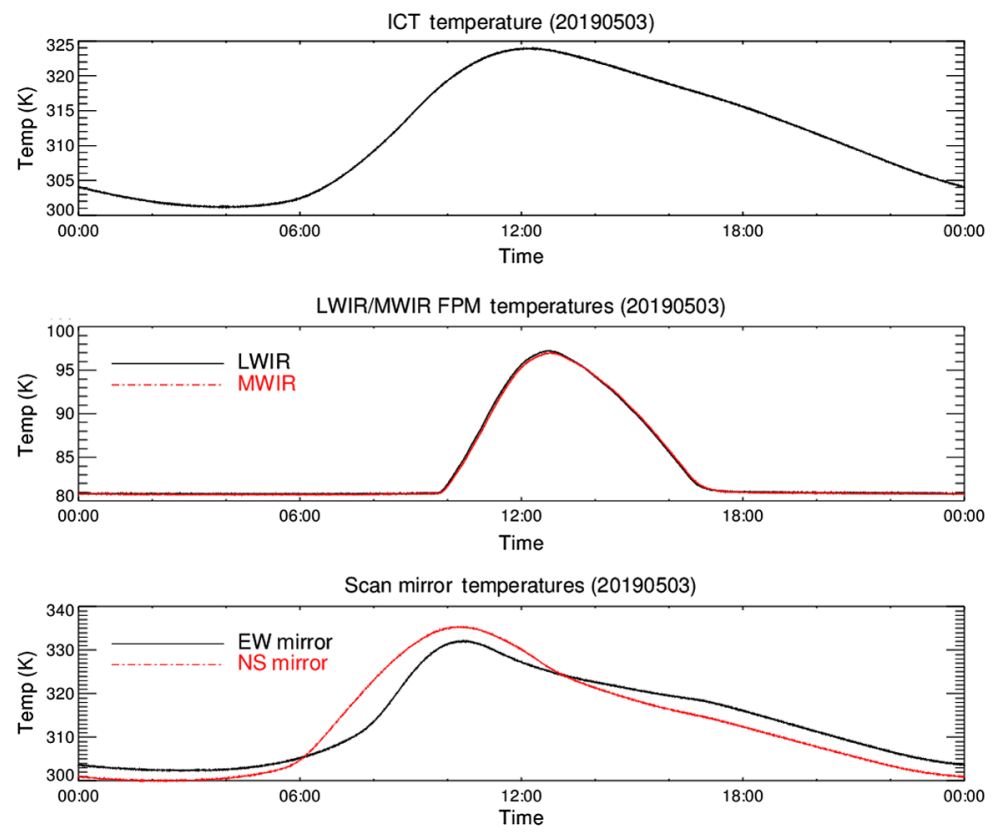

Fig. 1 The ICT, FPM, and scan mirror temperatures of GOES-17 ABI for May 3, 2019.

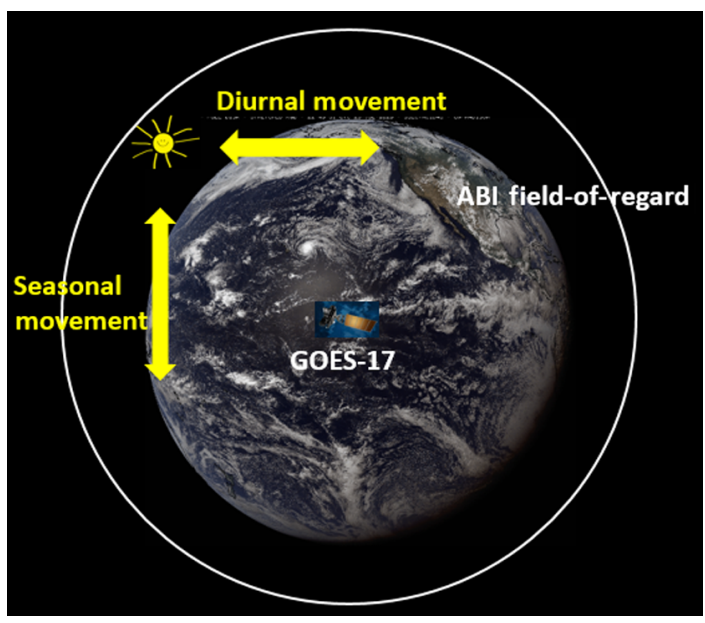

Fig. 2 A sketch illustrates the reception of the solar light when the Sun is at the opposite side of the Earth to GOES-17 ABI (aka local nighttime). ABI is situated above equator and faces into the paper. North is up. The true-color RGB ABI FD image is downloaded from Ref. 28.

the instrument is less intensive. Figure 3 shows the daily maxima and minima of the LWIR FPM temperatures from August 1, 2018, when the GOES-17 ABI PLPT started to December 15, 2019: while the daily minimal FPM temperatures can always maintain at $81 \mathrm{~K}$ throughout this period, the daily maximum temperatures exhibit four peaks corresponding to the pre-eclipse and posteclipse seasons and four valleys corresponding to solstice seasons and Equinox seasons in a year. The all-time high of FPM temperature was $\sim 108 \mathrm{~K}$ on April 14, 2019, corresponding to a diurnal variation of $27 \mathrm{~K}$. The three vertical dashed lines in the plot denote the satellite yaw flip maneuvers executed on September 24, 2018, March 27, 2019, and September 9, 2019, respectively, with the purpose of turning the ABI optical aperture away from the Sun to minimize solar load.

There are still ongoing efforts by L3Harris to reduce the heat inflow and suppress the FPM temperatures further, especially during their peak seasons. These efforts are not expected to alter their oscillation patterns, which means the IR imaging of GOES-17 ABI and its calibration will 


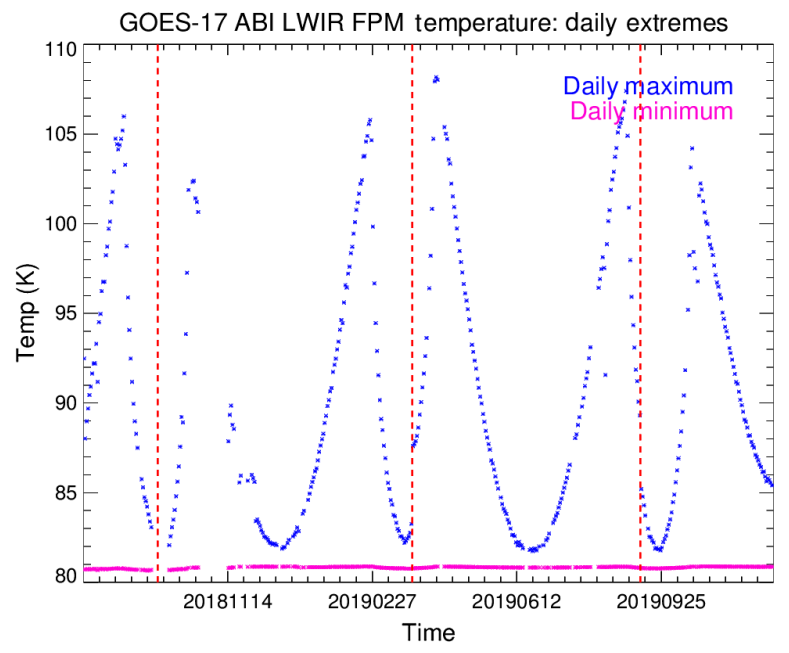

Fig. 3 The daily extremes of GOES-17 ABI LWIR FPM temperatures from August 1, 2018, to December 15, 2019. The vertical red dashed lines denote the time of satellite yaw flip.

be conducted under the same dynamic thermal conditions for mission life. The LHP anomaly also causes the fluctuation of the VNIR FPM temperatures. Its impact to VNIR calibration, which is less significant compared to IR, is under investigation.

\subsection{Deterioration of IR Performance due to the LHP Anomaly}

ABI IR detectors are manufactured in mercury cadmium telluride $\left(\mathrm{Hg}_{1-x} \mathrm{Cd}_{x} \mathrm{Te}\right)$ with composition $x$ varying with bands. ${ }^{20}$ The performance of such a detector is closely associated with its operating temperature or specifically the FPM temperature for ABI. The elevated FPM temperature baseline of $81 \mathrm{~K}$ for GOES-17 ABI suggests that its IR imaging quality will degrade as compared with the design and its GOES-16 counterpart. The degradation will be even worse at higher FPM temperatures. In general, the IR performance oscillates daily and seasonally as a result of FPM temperature fluctuation. Similar fluctuation has occurred for MODIS aboard the Aqua satellite at a much smaller scale of as much as $0.7 \mathrm{~K}$, and the induced negative impact on IR L1b was assessed and concluded to be mostly negligible. ${ }^{21,22}$ The magnitude of the FPM temperature fluctuation for ABI is unprecedented and results in more severe degradation to its L1b radiance products. The assessment of its impact is more complicated as well.

Among the artifacts induced by the anomalous FPM temperature, the increase of the dark offset signal with the FPM temperature has the greatest impact on the L1b products. For $\mathrm{ABI}$, the detector dark offset is measured at each SL in digital counts as $x_{\text {space }}$. Figure 4 shows how $x_{\text {space }}$ of several arbitrarily selected detectors of bands 8 to 16 vary within a day. The FPM temperature on April 14, 2019, is higher, so the saturation ranges are wider. From Fig. 1, it is clear that the trending of $x_{\text {space }}$ follows the trending of FPM temperature. The trending patterns are band dependent with small in-band detector-to-detector variation in magnitudes. The dynamic range of a detector shrinks with the increase of $x_{\text {space }}$. When $x_{\text {space, }}$, 14-bit integer, reaches its saturation level of $2^{14}-1=16,383$, the detector saturates completely and cannot capture any Earth image. It should be noted that the detectors for ABI bands 13 to 16 are integrated "down" at A/D conversion, which means smaller counts of $x$ correspond to larger radiance signals. The saturation level of these detectors is then zero. For the same reason, the gain coefficient $m$ calculated by Eq. 1 for bands 13 to 16 is negative, so this feature will be referred to as "negative gain."

The system-level gains of ABI detectors are configurable per individual band by altering the detector gain in analog amplification and the integration time. To alleviate the saturation, all detectors of bands 8 to 16 are now operated at two distinct gain sets. Each gain set is composed of a set of band-dependent configuration parameters gain set I is designed to maximize performance at the new temperature baseline of $81 \mathrm{~K}$; a different set, gain set III following the convention from L3Harris, reduces the detector gain and is designed to run at elevated and fluctuating FPM temperatures to suppress the dark offset signal to maximize the IR data 
Wang et al.: On-orbit calibration and characterization of GOES-17 ABI IR bands...

(a)
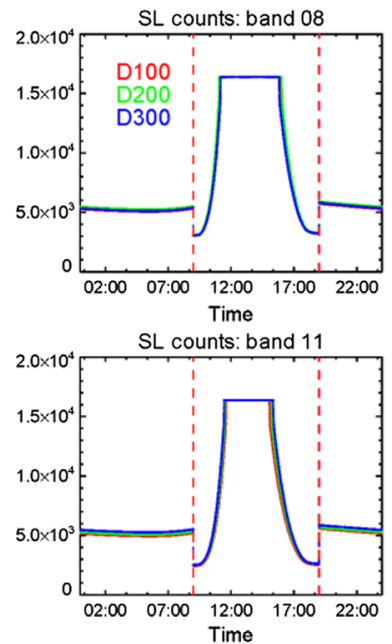

SL counts: band 14

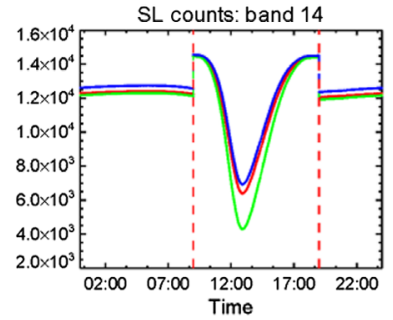

(b)
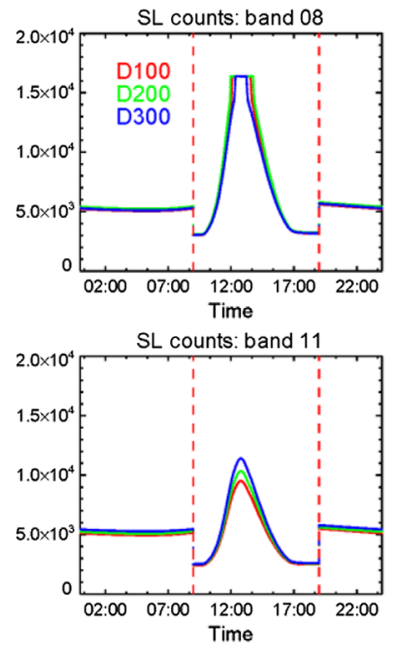

SL counts: band 14

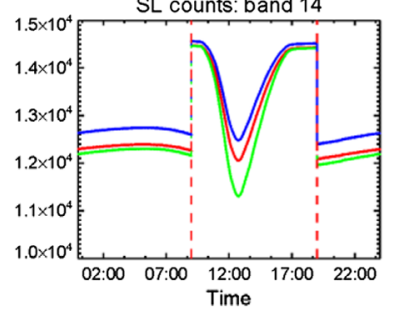

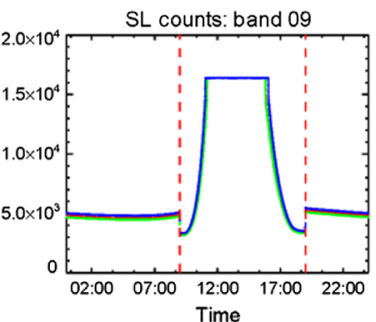

SL counts: band 12
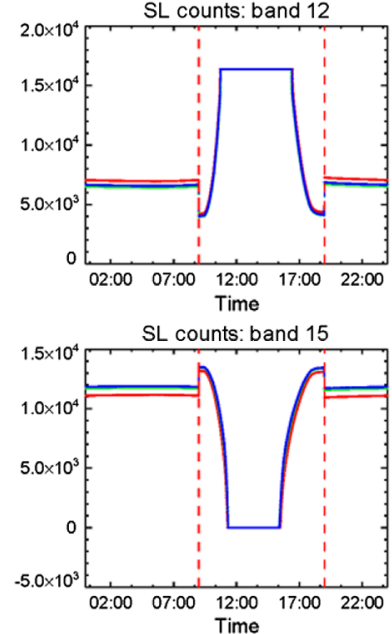

SL counts: band 09

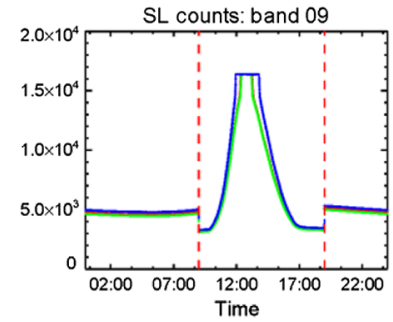

SL counts: band 12
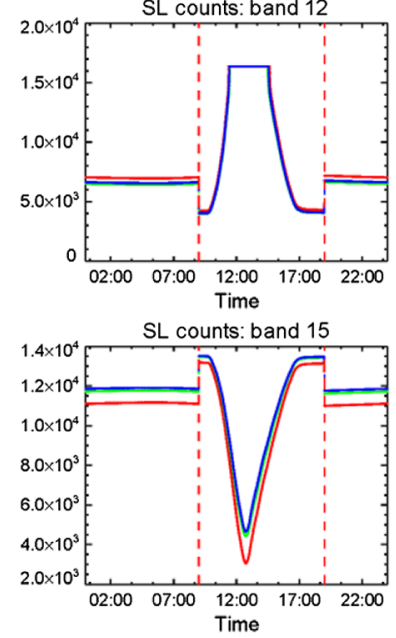
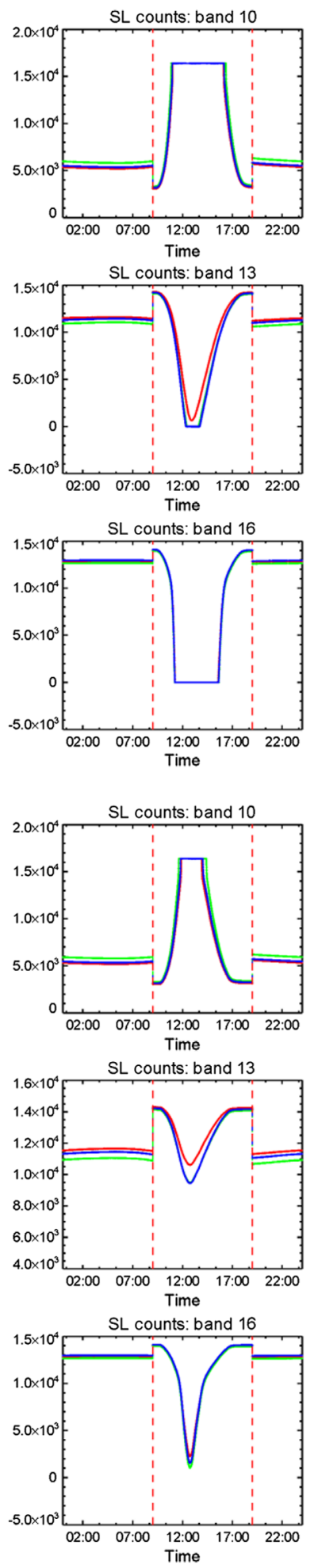

Fig. 4 The daily profile of SL counts of GOES-17 ABI IR detectors during (a) May 3, 2019, and (b) April 14, 2019. The peak LWIR temperatures are 98 and $108 \mathrm{~K}$, respectively. The vertical red dashed lines represent the time of gain switch.

availability. An intermediate gain set II was developed but later eliminated from operation. The detectors switch between the two gain sets on a daily basis for most times of the year when the daily maximum FPM temperature exceeds $85 \mathrm{~K}$. Otherwise, the gain switch is disabled. The time of switch is currently set at 0900 UTC and 1900 UTC but is adjustable to 0830 UTC and 1930 
Wang et al.: On-orbit calibration and characterization of GOES-17 ABI IR bands...

Table 2 GOES-17 gain switch time summary.

\begin{tabular}{llcc}
\hline \hline Starting date & \multicolumn{1}{c}{ Ending date } & Gain set I-III (UTC) & Gain set III-I (UTC) \\
\hline November 13, 2018 & December 6, 2018 & 0830 & 1930 \\
December 6, 2018 & January 18, 2019 & - & - \\
January 18, 2019 & March 20, 2019 & 0830 & 1930 \\
March 20, 2019 & May 28, 2019 & 0900 & 1900 \\
May 28, 2019 & July 16, 2019 & - & - \\
July 16, 2019 & September 10, 2019 & 0900 & 1900 \\
September 10, 2019 & October 6, 2019 & - & - \\
October 6, 2019 & March 18, 2020 & 0830 & 1930 \\
March 18, 2020 & March 25, 2020 & - & - \\
March 25, 2020 & June 1, 2020 & 0900 & 1900 \\
\hline \hline
\end{tabular}

Note: Hyphen (-) indicates the gain switch is disabled. The IR detectors operate at gain set I only.

UTC. The adjustment is semiannual after each yaw flip to maximize the operational time period of gain set I. Table 2 summarizes the gain switch time of GOES-17 since its orbital drift to GOES-West position. The two vertical red dashed lines in Fig. 4 represents the gain switch time for the day. The time period bracketed by the two lines is the gain set III period and the rest of the day is the gain set I period. It can be seen from the plot that the magnitude of $x_{\text {space }}$ is brought down upon the activation of gain set III and up again by its deactivation. This, together with the lower gain value itself, extends the dynamic range of a detector dramatically. Even with the dual gain setup, detectors of bands 8 to 10 and 12 still get saturated during the hottest period of time on May 3, 2019. The saturation is worse at higher FPM temperatures, and all IR bands with center wavelengths longer than $4 \mu \mathrm{m}$, except band 14 and some detectors of band 13, will saturate at the all-time temperature peak of $108 \mathrm{~K}$. Many band 13 detectors saturate near peak temperature, and there is an ongoing test to reduce the detector gain of band 13 to make its data consistently available for mission life.

In-depth analysis of the FPM temperature impacts on the key IR performance parameters will be provided in the following sections. Unlike other bands, band $7(3.9 \mu \mathrm{m})$, a low-gain fire detection band with a much larger dynamic range of input radiance, is insensitive to the FPM temperature variation, so its results from the analysis will not be covered in this paper.

\section{Calibration Bias due to Drifting FPM Temperature: Root Cause and Mitigation}

Whenever the FPM temperatures start to drift from the $81 \mathrm{~K}$ baseline, calibration bias is brought into L1b radiance products for GOES-17 ABI IR bands. Other than the fluctuating FPM temperature itself, this instrument-unique anomaly is associated with ABI's scanning mechanism, which is introduced in detail below.

\subsection{Introduction of $A B I$ Scan Timeline}

An ABI "scene" defines the region of interest or target that is to be scanned. ABI views various scenes by the rotation of its NS and EW scan mirrors, simultaneously if needed. Other than the three types of EV scenes of FD, CONUS, and MESO, there are other predefined scenes imaging internal and external targets including calibrators. A scan timeline, or scan mode, is a schedule that defines when each selected scene is viewed within a fixed time period. ABI has three primary scan modes: 5-min mode 4 collects just the FD while 15-min mode 3 and 10-min mode 6 
(a)

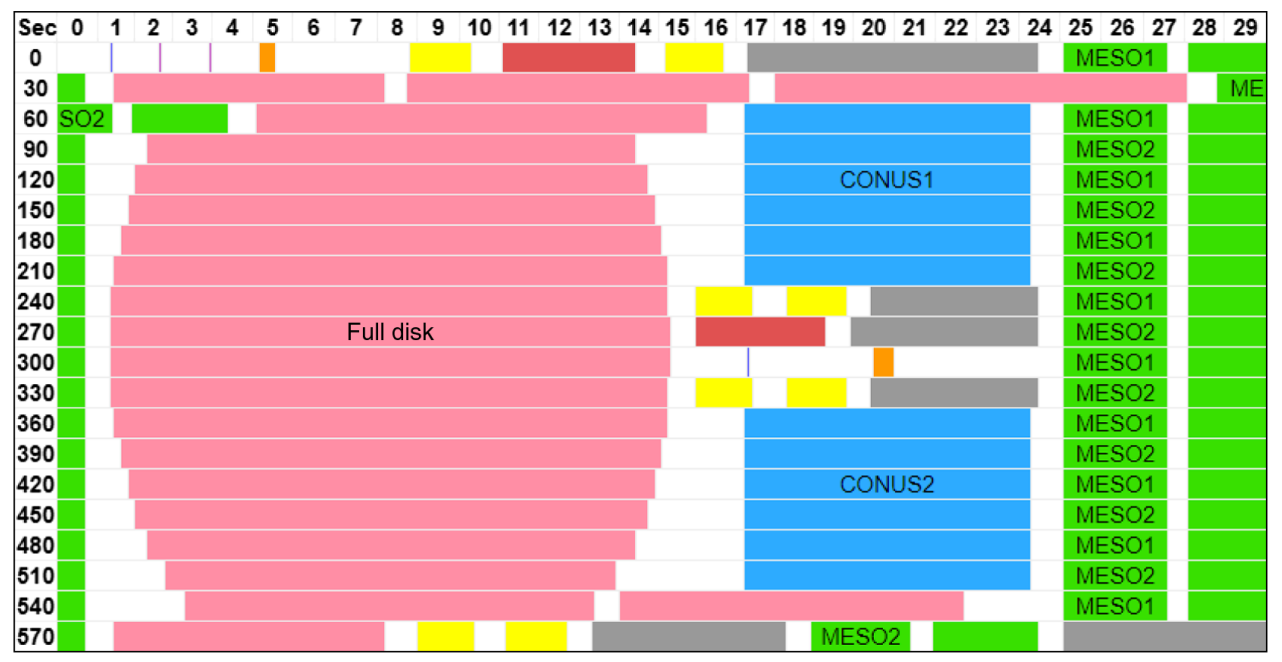

(b)

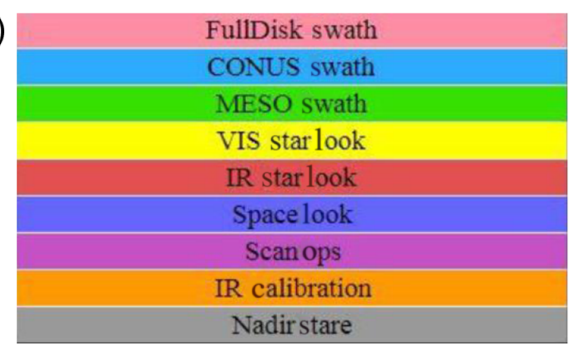

Fig. 5 The diagram of (a) scan mode 6M currently operational for GOES-17 ABI and (b) its scene legend. ${ }^{29}$

provide more flexible storm watch capability by collecting CONUS and MESO together. Mode 3 had been the default operational mode for both GOES-16 and GOES-17 ABIs until April 2, 2019, when mode 6, also known as "10-min flex mode," became the default. The mode 6 timeline has been evolving to address various instrumentation issues, in particular for GOES-17 to address the LHP anomaly. Figure 5 shows the diagram of mode $6 \mathrm{M}$ currently operational for GOES-17 ABI. A similar mode 6A is operational for GOES-16 ABI. Each row in the diagram represents a 30-s scan line with its starting time within the timeline, in units of seconds, provided in the beginning. Both the EW and NS scan mirrors could move within a scan line to view the defined scenes so a scan line shall not be interpreted as a scan swath for a cross-track scanner. The whole timeline is 10-min long, consisting of 20 scan lines. Occasionally, one scan line may consist of two FD scan swaths in the EW direction, but most of the scan lines consist of just one. One mode 6 timeline provides one FD scene in 22 swaths, two CONUS scenes in 6 swaths each, and 20 MESO scenes in two swaths each.

Within each timeline, the EV scenes are interleaved with necessary scenes for radiometric and spatial calibrations. The ICT calibration scene (ICT look) is imaged every 5 min in mode $6 \mathrm{M}$ at the beginning and in the middle of the timeline to calculate the gain coefficient $m$. The SL scene to measure detector background offset is taken for each FD swath, as well as each ICT look. For simplicity, not all of the SL scenes are explicitly plotted in this timeline diagram of Fig. 5. For mode 6A in GOES-16, there is only one ICT look at the beginning of the timeline, which means ICT calibration is performed every $10 \mathrm{~min}$. The intervals between the gain calculation and between SL measurements are relatively large. By contrast, the IR calibration of MODIS and VIIRS is conducted for every scan of 1.478 and $1.779 \mathrm{~s}$, respectively, frequently enough to capture any potential detector instability.

\subsection{Calibration Bias Induced by Drifting FPM Temperature}

To accurately calculate $m$ or $L_{\mathrm{EV}}$, the input variables of Eqs. (1) and (2) must be determined exactly for the time of $m$ or $L_{\mathrm{EV}}$ measurements. However, for GOES-17 ABI IR bands 8 to 16 , 

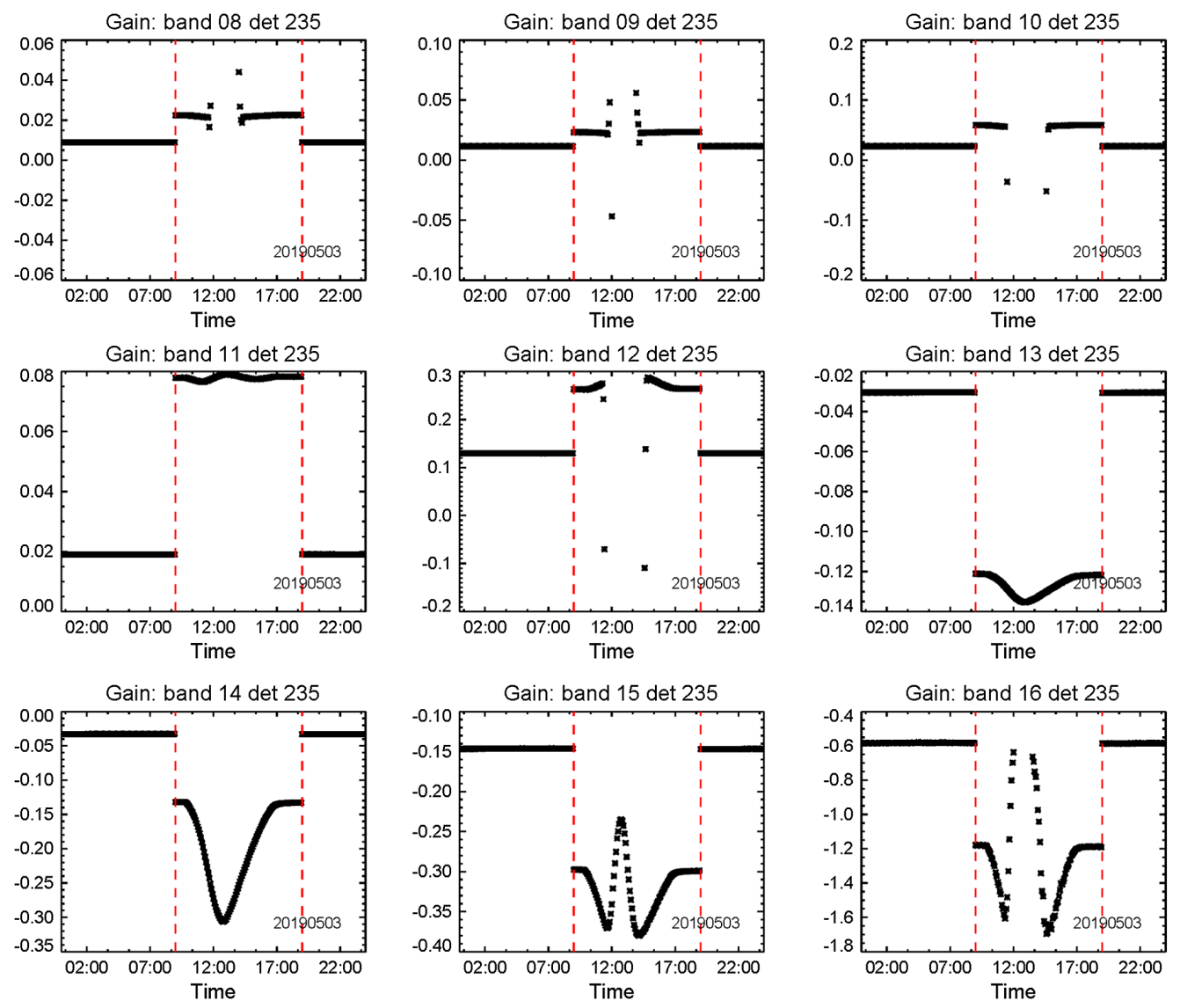

Fig. 6 The gain coefficient trending of typical GOES-17 ABI IR detectors for May 3, 2019. The vertical red dashed lines represent the time of gain switch.

the offset signal $x_{\text {space }}$ changes rapidly with its FPM temperature during the hot period of the day, as shown in Fig. 4. Since it is measured ahead of the ICT look or the EV scene, the measurement does not accurately reflect its true value at the time of the ICT look or EV sample. In addition, the detector gain of IR detectors also changes with the FPM temperatures, as shown in Fig. 6, which plots the gain coefficient $m$ of the typical detector \#235 in each band for May 3, 2019. For some bands, bands 13 to 16 in particular, the trends of $m$ clearly follow the trend of FPM temperatures. When $m$ is dynamic, bias will be introduced into the calibrated radiance when the measurement of $m$ is well ahead of the EV scene. Because of detector saturation during the hottest period of a day, gain coefficients are not available for several bands such as 8 to 10,12, and 16. This is reflected in Fig. 6 as gaps in the trending.

The overall bias with the FPM temperature fluctuation can be subdivided into three components.

1. The SL measurement $x_{\text {space }}$ for the EV scene is taken at the limb of each FD swath, either before or after depending on the solar illumination condition. According to Fig. 5, the time lag between the SL measurement and the farthest EV samples can be as long as 13.5, 21.5, or $28.5 \mathrm{~s}$ for FD, CONUS, and MESO scenes, respectively. The $x_{\text {space }}$ of IR detectors can change by dozens during the period, causing extensive L1b bias in all three types of EV scenes. For any given band and detector, the magnitudes of the bias monotonically increase with the time lag, reflected in the L1b image as banding along the EW direction. The largest bias of this type is for MESO scenes, which is measured at the end of each scan swath. Within each scene type, the largest bias is for the swath that scans the equator when the time lag between the measurements of $x_{\text {space }}$ and the EV sample is the longest. The bias is positive when the FPM temperature is rising and negative when the FPM temperature is falling. 
2. The gain measurement $m$ is taken at the beginning of the timeline and in the middle of the timeline after the equatorial swath and thus differs from the true gain at the time of the EV samples. The magnitudes of the bias in radiance are proportional to the time lag, notably reflected in the L1b image as banding along the NS direction. The largest bias of this type is at the equator and the south pole region when the time lag is the longest. The bias is negative when the FPM temperature is rising and positive when the FPM temperature is falling.

3. The SL measurement $x_{\text {space }}$ for ICT calibration is taken $\sim 4 \mathrm{~s}$ prior to the ICT look, creating a bias in the calculation of $m$. The bias is then carried into the calibration of $\mathrm{EV}$ images of the next $5 \mathrm{~min}$. This type of bias is similar to bias type 1 in its analysis but is less significant.

In recent years, the increasing data availability of remote sensing instruments with radiometric calibration capability makes intersensor comparison a convenient and powerful tool for assessing the accuracy and monitoring the stability of their calibrations. The calibration bias of GOES-17 ABI is assessed by comparing its L1b radiance with other geostationary satellites, such as GOES-16 ABI, based on the observation of collocated Earth sites. ${ }^{23}$ The site selected is centered on the equator at $106 \mathrm{deg} \mathrm{W}$, approximately the midpoint of the two satellites, to minimize the radiometric discrepancy induced by the difference of view geometry. The comparison results are regularly generated and posted online on the CWG website. ${ }^{24}$ A sample result using L1b FD images as input is shown in Fig. 7 in green symbols for May 1, 2019, at a peak LWIR FPM temperature of about $97 \mathrm{~K}$. The GOES-16 ABI IR calibration has been proved stable at a
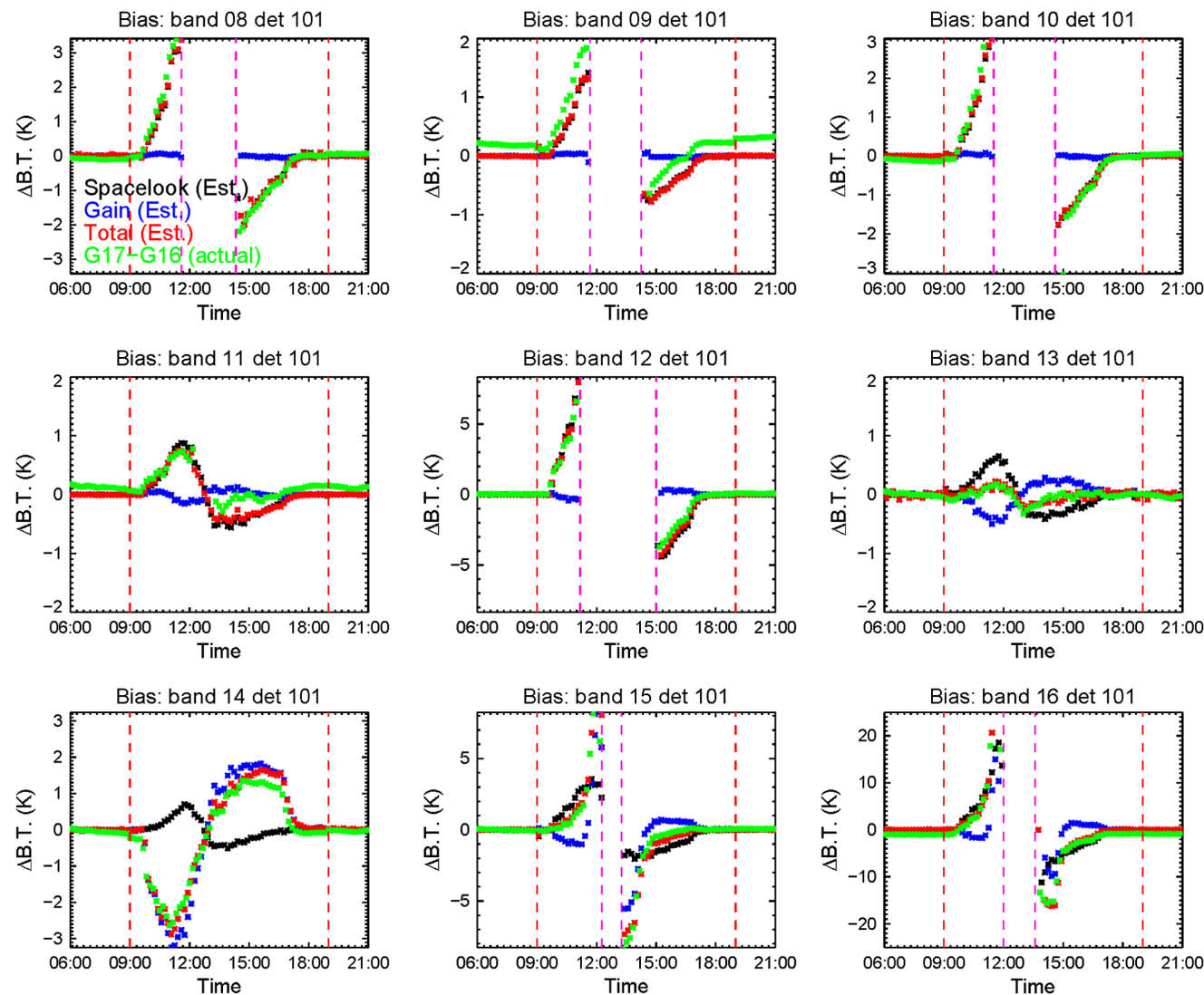

Fig. 7 The difference between the calibrated BT of GOES-16/GOES-17 ABIs on May 1, 2019, is compared with the estimated GOES-17 calibration bias induced by floating FPM temperature: black: estimated bias induced by SL counts variation; blue: estimated bias induced by gain variation; red: estimated total bias; green: offset observed from GOES-16/GOES-17 comparison. The red vertical lines are the gain switch time. The purple vertical lines bracket the period of ICT look saturation. 
magnitude of $<0.2 \mathrm{~K}$ through its intersensor comparison with well-calibrated hyperspectral sounders IASI and CrIS, ${ }^{25}$ which will be the calibration bias of GOES-17 ABI that contributes to the majority of the GOES-17/GOES-16 calibration offset.

On the other hand, with the $x_{\text {space }}, x_{\mathrm{ICT}}$, and $m$ output in INST-CAL files, together with the time of their measurements, the actual values of these parameters at any time within a timeline can be estimated by temporal interpolation. Then, instead of using the $x_{\text {space }}, x_{\mathrm{ICT}}$, and $m$ measured prior to the EV image as the original GPA does, the interpolated parameters are brought into the calibration [Eq. (2)] to recompute radiance. The difference between the recomputed radiance and the original radiance is the estimated calibration bias, which is then converted into BT bias, as shown in Fig. 7. In the figure, blue symbols represent the bias component induced by gain variation with FPM temperature, black symbols represent the bias component induced by SL counts variation with FPM temperature, and red symbols represent the combined bias. The two vertical red dashed lines represent timing of gain set I/III switch at 0900 UTC and 1900 UTC for the date. The two vertical purple lines bracket the time period when detector saturation occurs, and thus the calibration is completely unusable so the data within can be ignored from the analysis. As seen, the combined bias estimation overall matches the observed bias. For band 9,11 , and 16, there are temporally invariant offsets between the estimated and actual bias. They are likely caused by the inadequacy of the current GOES-16/GOES-17 intersensor comparison approach, in which the differences between the SRFs of the matching bands of the two ABIs and the viewing geometry are not corrected. Overall, the results confirm that the majority of the GOES-17 ABI calibration bias observed during the hot period of the day is caused by the variation of FPM temperatures. The patterns of the bias are mostly band dependent with insignificant detector dependency. For most of the IR bands, the bias induced by SL counts variation with FPM temperature is the dominant component. The only exception is for band 14, in which the bias component induced by gain variation with FPM temperature dominates.

There are certain limitations to the selection of collocated sites for GOES-16/GOES-17 comparison, so currently this approach can only assess the temporal changes of the calibration bias at a static position within a timeline in time domain or within an L1b image in spatial domain. The spatial distribution of the bias within a timeline or an L1b thus cannot be demonstrated directly. However, the distribution can be largely estimated based on the above analysis of the bias source and referencing the bias at the equator in Fig. 7.

Another illustration of the calibration bias induced by the variation of $x_{\text {space }}$ with the FPM temperature is shown in Fig. 8. On September 10, 2018, for the first time, all four MESO scenes of GOES-16 and GOES-17 ABIs observed the same EV spot on top of Hurricane Florence. The mean radiance is calculated per MESO scene acquired every $30 \mathrm{~s}$ and trended from $1200 \mathrm{UTC}$ to 1218 UTC in the plot. Their MESO-to-MESO variation is a measure of calibration repeatability and is expected to be small. While the results for GOES-16 ABI are reasonably stable, for GOES-17 ABI, the mean radiance increases by $~ 1 \%$ for the two MESO scenes of 12:05:30 UTC and 12:12:00 UTC. Band 10 results are shown here, while the same increases were seen in most other bands. At the time when these MESO scenes were taken, GOES-17 ABI was running the mode 3 timeline of 15 -min FD. It was found that the two MESO scenes with increased radiances are the ones following the extra ICT calibrations and their associated

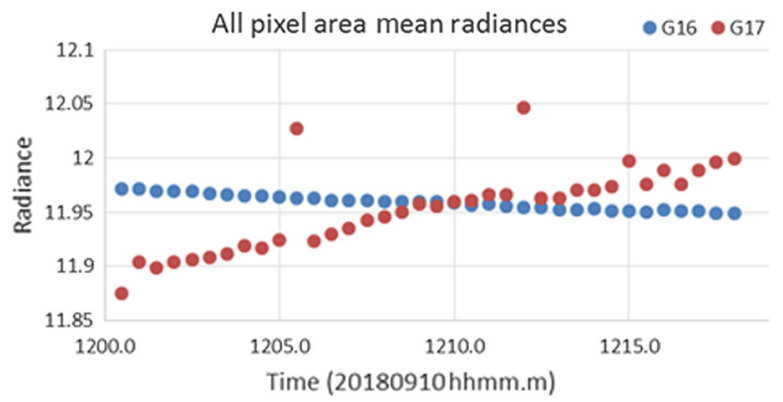

Fig. 8 The mean radiance of $A B I$ band 10 MESO images captured for Hurricane Florence within a short 18-min period in September 2018. 
SLs. For the rest of the MESO scenes, $x_{\text {space }}$ was measured at the beginning of the FD scene. The ABI scene is calibrated using the immediate measurement of $x_{\text {space }}$, regardless of the purpose of its addition. Therefore, the two MESO scenes are calibrated with the $x_{\text {space }}$ that has a different relative offset relative to the truth due to its relative change in timing combined with the drifting temperatures. Their calibration bias is thus different from the others, making them outliers in radiance trending. This observation was one of the earliest signs of the existence of calibration bias caused by SL counts variation that triggered our investigation on the matter.

\subsection{Bias Mitigation by pCal Algorithm}

Limited by the ABI scanning mechanism, it is practically impossible to dramatically increase the sampling interval of $x_{\text {space }}$ or $m$ to better track their variation with the FPM temperature. Instead, a revision of the IR calibration algorithm was proposed to mitigate its impact. Rather than directly using the immediate measurements, $x_{\text {space }}$ and $m$ applied to the calibration Eqs. (1) and (2) are projected through the temporal extrapolation of the previous two measurements to account for their drift with the FPM temperature. The methodology is realized by the pCal algorithm developed by L3Harris and the GOES-R Program. ${ }^{12}$ The extrapolation projection contains three separate elements to separately address the above three types of bias.

1. Projecting the SL at the time of detector EV sample.

2. Projecting the gain at the time of detector EV sample.

3. Projecting the SL at the time of gain coefficient calculation.

This algorithm assumes the temporal changes of the FPM temperature, $x_{\text {space }}$, and $m$ are gradual, so their extrapolations can well approximate the actual values. The choice of using linear extrapolation is somewhat empirical, and its accuracy is validated by the examination of the real data. Any singular point in the trends of $x_{\text {space }}$ and $m$, if it exists, may yield a very large error in their projection, and the error would be magnified further with higher-order extrapolation schemes.

The pCal algorithm can be switched on/off in the GPA. A key component of the pCal algorithm is the determination of its on/off time. During the period of the day when the FPM temperature is stable at $81 \mathrm{~K}$, the default calibration algorithm without $\mathrm{pCal}$ is adequate. Using the extrapolation of $x_{\text {space }}$ and $m$ during the period essentially amplifies the detector instability and noise. Therefore, $\mathrm{pCal}$ should be engaged only when the FPM temperature is drifting. In particular, when the gain set I/III switch is enabled, pCal cannot be active at the time of gain set switch. This is because the switch drastically changes the values of $x_{\text {space }}$ and $m$, as shown in Figs. 4 and 6. The extrapolated values using both gain set I and gain set III values will be off the truth completely. In the current algorithm, $\mathrm{pCal}$ is turned on or off every day by comparing the measured FPM temperature with the FPM temperature thresholds saved as a configurable LUT: $\mathrm{pCal}$ is on when the FPM temperature is above the threshold and off otherwise. The thresholds are separately set for MWIR and LWIR FPMs. As a result, the pCal on/off time for bands 8 to 11 differs from that for bands 12 to 16 . Because the start and end time of the FPM temperature drift is dynamic, the thresholds should be periodically adjusted to keep up pace with the seasonal oscillation of the FPM temperatures.

The pCal algorithm was tested in the integration and test environment (ITE), a part in the GS for algorithm development testing purposes, from June 16, 2019, to July 24, 2019, to ensure its software implementation correctness. Both the L1b produced by ITE with the pCal algorithm implemented and the corresponding L1b produced by operational environment (OE) without pCal algorithm implemented are compared with the collocated GOES-16 ABI L1b to verify the bias reduction: the environmental settings of ITE and OE are otherwise identical except for pCal, so any difference in GOES-16/GOES-17 comparison results between ITE and OE is from the $\mathrm{pCal}$ algorithm. Another goal of the test is to optimize the associated operational parameters, in particular the FPM temperature thresholds to turn on/off pCal.

The goal of the test was achieved: the bias induced by fluctuating FPM temperature is effectively reduced. One sample result is shown in Fig. 9. For this day, pCal was on from 1030 UTC to 1620 UTC for bands 8 to 11 and from 0850 UTC to 1700 UTC for bands 12 to 16 . With pCal 

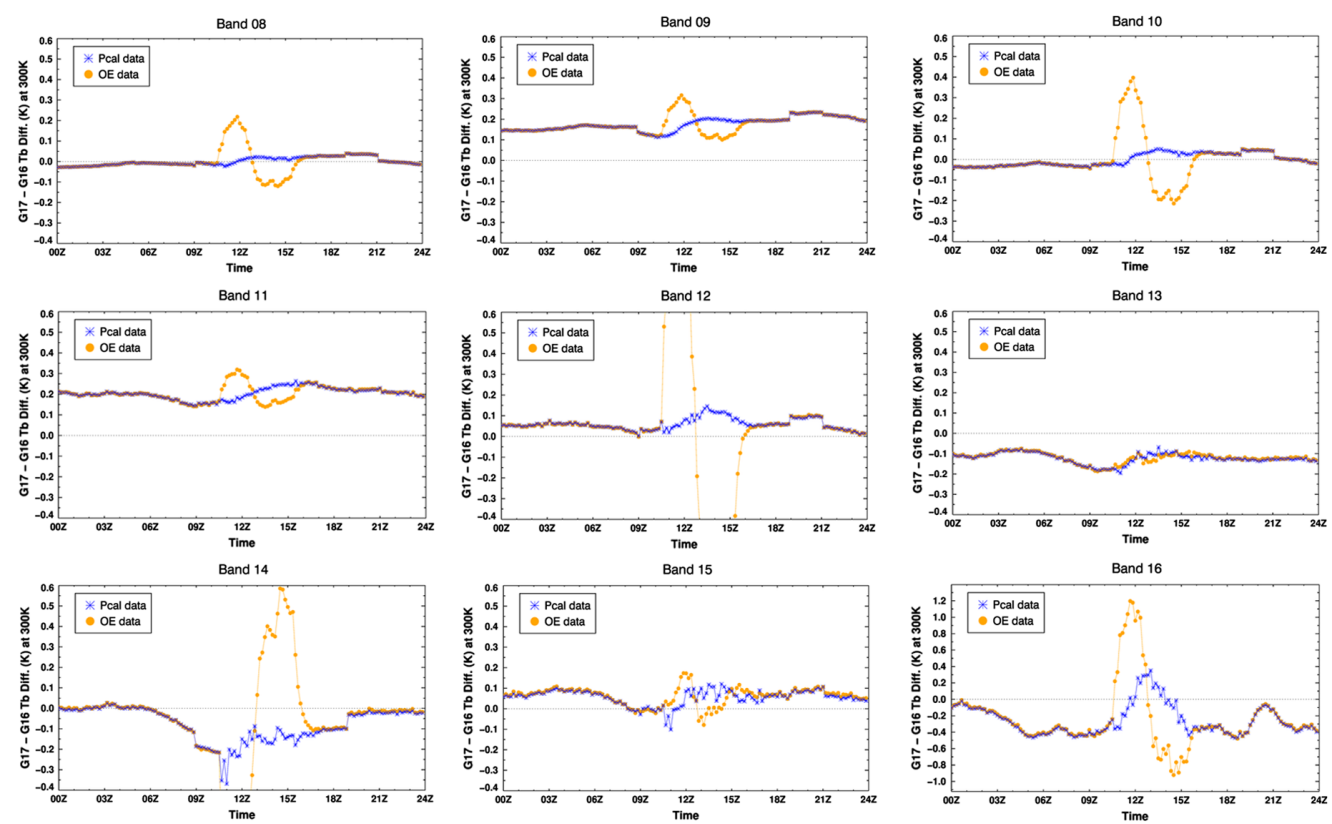

Fig. 9 GOES-16/GOES-17 L1b comparison of July 24, 2019: GOES-17 L1b generated in OE is calibrated without pCal algorithm and L1b generated in ITE is calibrated with pCal algorithm.

enabled in the ITE and not implemented in the OE, the comparison of GOES-17 OE and GOES16 exhibits notably large bias, while the comparison of GOES-17 ITE and GOES-16 yields much reduced bias. For the rest of the day when pCal is off in the ITE, the ITE and OE results are identical. The FPM temperature thresholds to turn on/off $\mathrm{pCal}$ were also optimized for concurrent FPM temperatures and predicted for the future. After the test, $\mathrm{pCal}$ was formally installed into the OE for operation on July 25, 2019. There have been ongoing efforts to further optimize the $\mathrm{pCal}$ on/off time, including a strategy developed by the GOES-R Product Readiness and Operations (PRO) team to trigger the switch by wall-clock time each day.

With pCal, even though extrapolation of $x_{\text {space }}$ and $m$ in the pCal algorithm approximates their actual values for EV samples much better than their immediate measurements, residual biases still exist and have been detected from the GOES-16/GOES-17 comparison. The residual can also be assessed offline from the calibration data. Since the offline assessment is a backprocessing, the $x_{\text {space }}$ and $m$ values both before and after the EV samples are available in INSTCAL. We assume that their temporal interpolation is an even better approximation of the actual values. Using the interpolation as reference, the residual bias from the extrapolation is estimated during the pCal test. One example is shown in Fig. 10. The large random spreads in the results of several bands, band 14 in particular, are not due to calibration bias but detector noise. Excluding the effect of noise, the residual bias is significantly smaller compared with the bias without $\mathrm{pCal}$, as shown in Fig. 7. The residual bias is mostly notable in bands 12 and 14, at a magnitude of $\sim 0.5 \mathrm{~K}$. The bias will be larger at higher FPM temperatures when detectors approach their saturation limit. This bias is due to a separate presaturation effect to be introduced later and not addressed by the pCal algorithm.

\section{Characterization of GOES-17 ABI IR Performance}

In addition to the calibration bias related to the FPM temperature drift, high FPM temperatures seriously degrade the IR performance of GOES-17 ABI in several other ways. The diagrammatic sketch in Fig. 11(a) shows how the IR performance changes with the FPM temperatures within a calendar day in UTC time. When the FPM temperatures are stable at the $81 \mathrm{~K}$ baseline (light green zone), the IR performance is good overall, as shown in Fig. 11(b), although the noise performance is still worse than for GOES-16 ABI. As the FPM temperatures increase, detector noise increases (dark green zone) until it exceeds the specification (gray zone). The dynamic range shrinks and calibration bias is induced. At even higher FPM temperatures (yellow zone), 

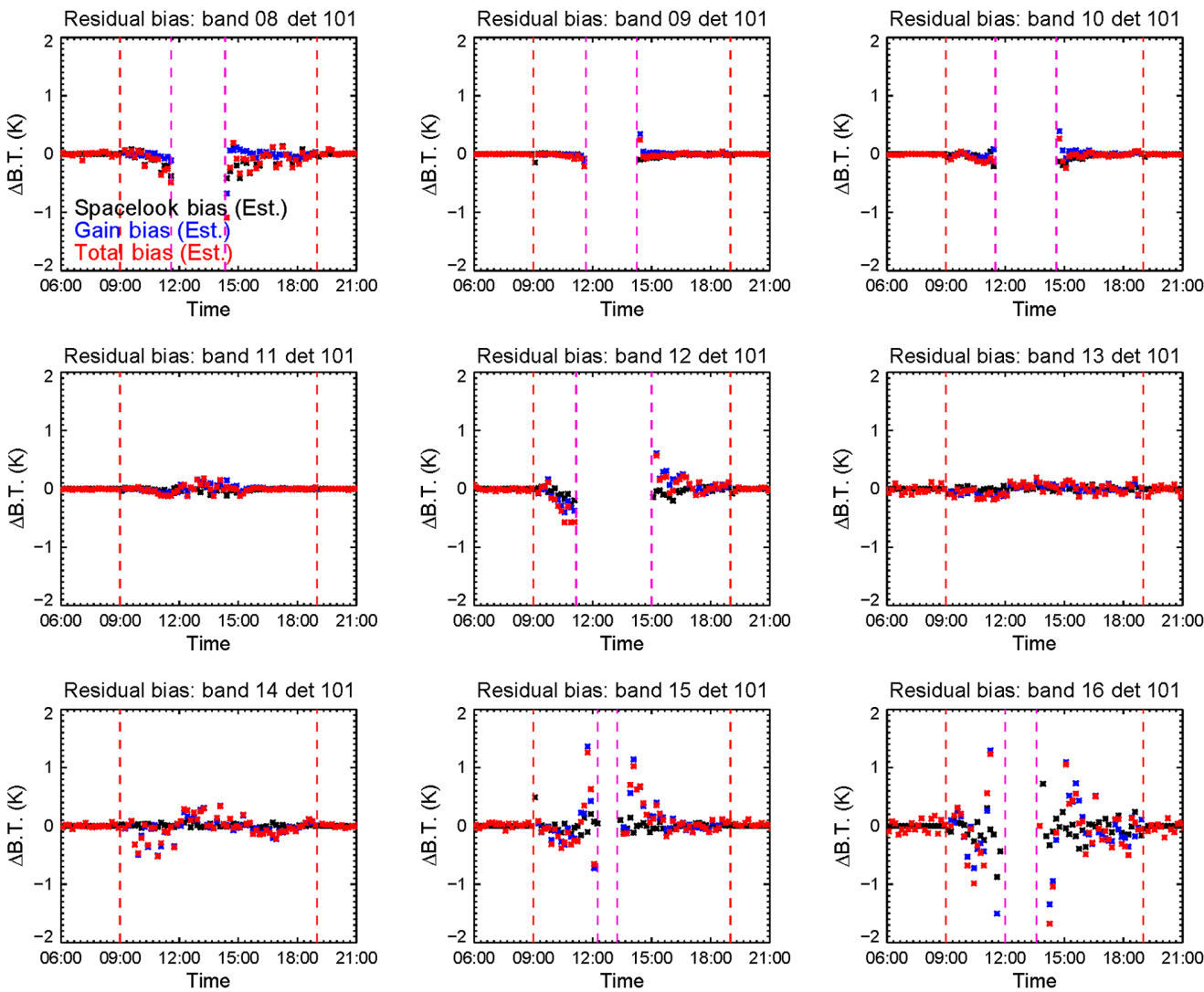

Fig. 10 The residual calibration bias of $\mathrm{pCal}$ algorithm estimated from the calibration data. Black: estimated bias induced by SL variation; blue: estimated bias induced by gain variation; red: estimated total bias. The red vertical lines are gain switch time. The purple vertical lines are the time of ICT saturation.

the detector's ICT look, $x_{\mathrm{ICT}}$, saturates, so the calculation of gain coefficient is erroneous, causing severe calibration bias shown as serious horizontal stripes in Fig. 11(c). When the FPM temperatures continue to rise, the detector' dark current, measured as $x_{\text {space}}$, saturates (red zone), so the detector cannot output any L1b radiances. Some detectors saturate first; the saturation of these detectors is reflected in L1b radiances as white stripes in Fig. 11(d). More and more detectors saturate at higher temperatures [Fig. 11(e)] until complete saturation. The sequence is reversed when the FPM temperatures move downward. As introduced above, the successful development and implementation of pCal nearly removes the bias due the FPM temperature variation, yet it does not resolve the other FPM temperature-related impacts.

The FPM temperature thresholds separating various performance zones are band and detector dependent, which means that different in-band detectors enter various performance zones at different times. When an individual detector enters a degraded performance zone first, the artifact is for this detector only and is reflected in L1b radiance images as stripes along the EW direction as the LOS of the detector moves in the direction by the rotation of the EW mirror. When more detectors enter the same performance zone, the quality of the whole L1b image degrades accordingly. Because of the seasonal oscillation of the FPM temperature, an IR band may not enter all of these performance zones every day. The IR performance at any time is strictly tied to the FPM temperature at the time and its comparison with these thresholds. Therefore, the assessment of these thresholds is essential to quantifying the GOES-17 ABI IR performance.

\subsection{IR Detector Saturation/Presaturation}

Figure 12 shows the relationship between the FPM temperature and the $x_{\text {space }}$ or $x_{\text {ICT }}$ for May 3, 2019, using the same input data as Fig. 4. The relationship on this day is typical throughout the 
(a)

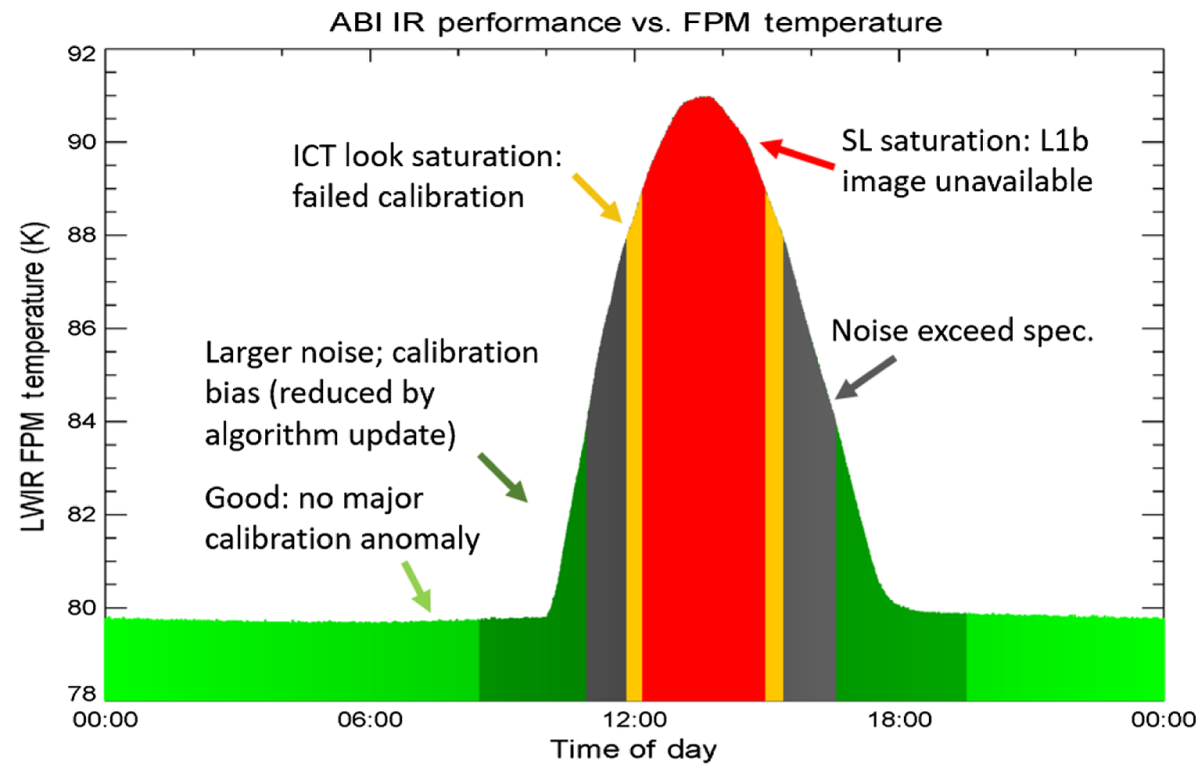

(b)

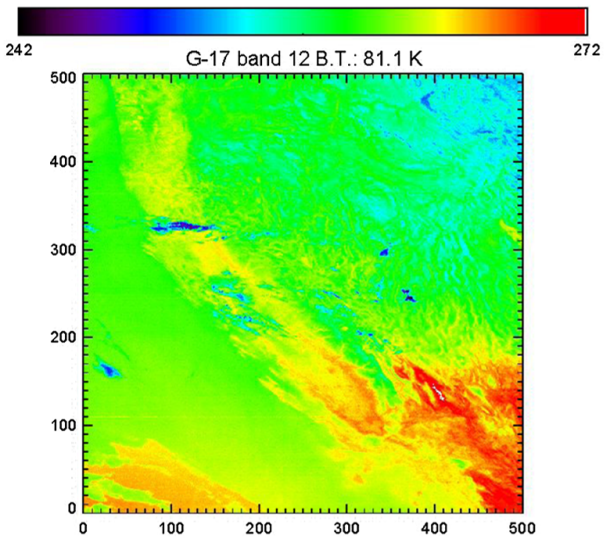

(d)

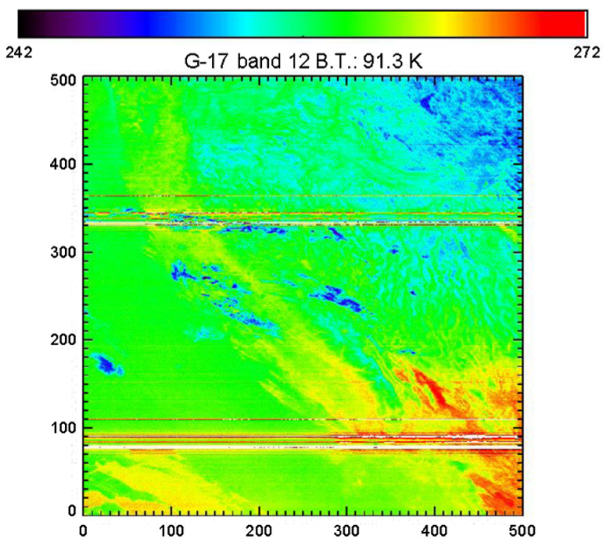

(c)

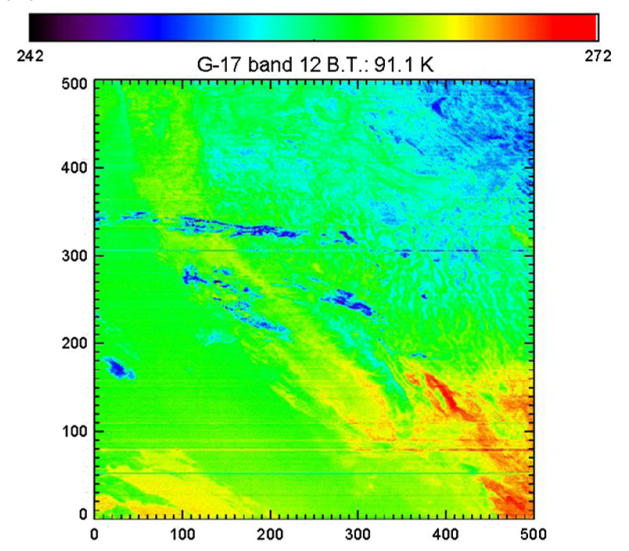

(e)

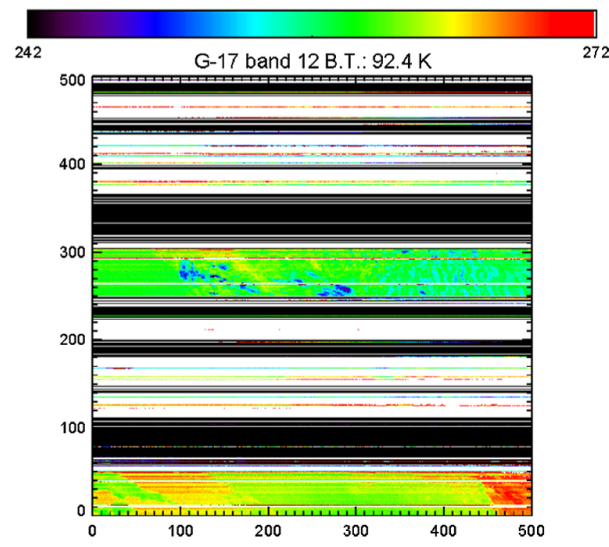

Fig. 11 (a) A sketch summarizing the dependence of ABI IR performance on the FPM temperatures. The MESOs from (b) to (e) demonstrate how $L 1 b$ qualities degrade with rising FPM temperatures for a period from 0930 UTC to 1045 UTC on August 28, 2019.

year. There are many more data points for $x_{\text {space }}$ than $x_{\text {ICT }}$ because the former is sampled every scan line of $30 \mathrm{~s}$ and the latter is calculated every $5 \mathrm{~min}$. In general, there is a monotonic relationship between the counts and temperatures before detector saturation. The curves for the rising and falling sides of the FPM temperatures deviate from each other, most notably for band 12 . 

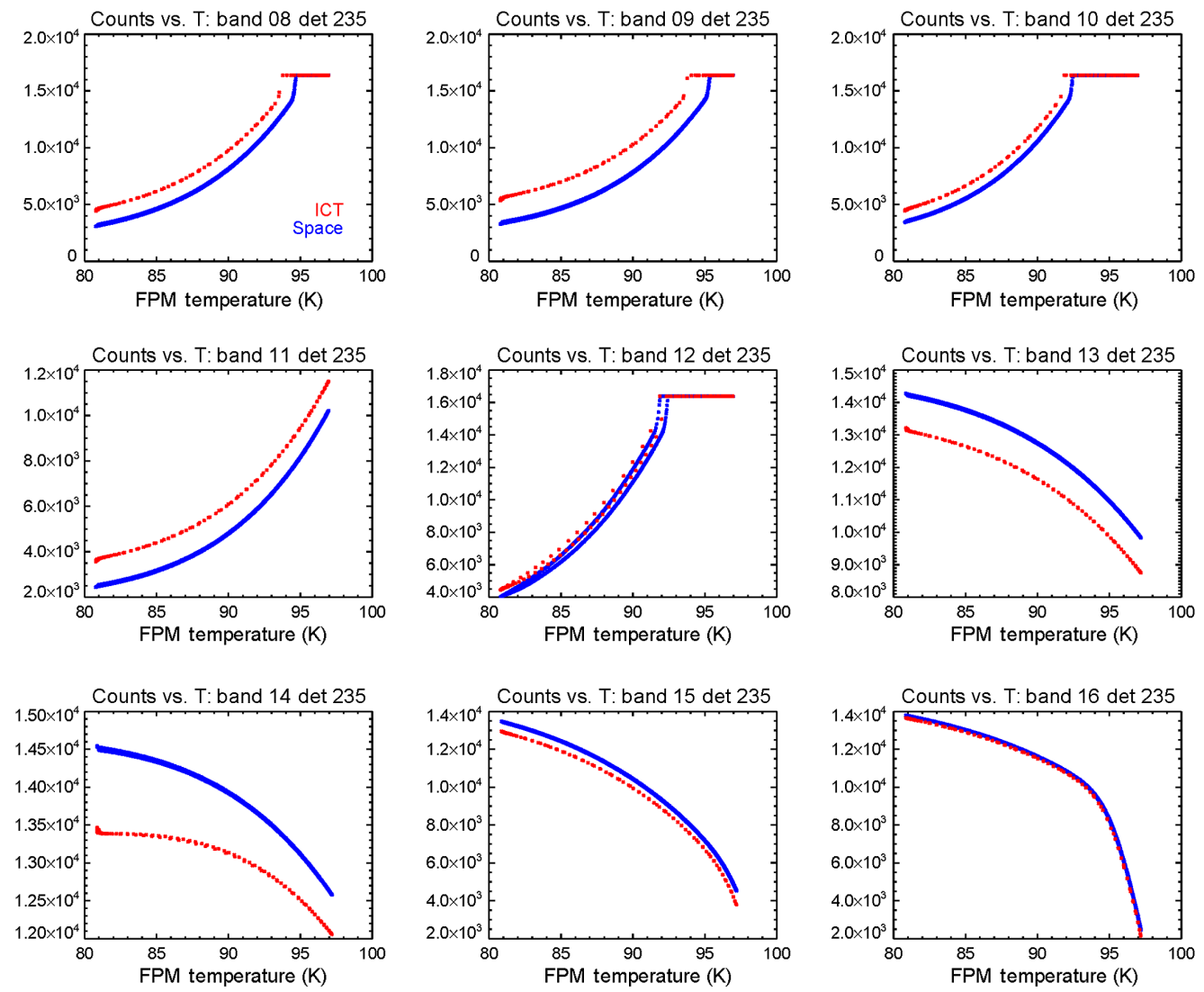

Fig. 12 The relationship between the FPM temperature and the detector counts at SL and ICT look for May 3, 2019.

The exact reason for this is still under investigation. Another noticeable feature is that, when the $x_{\text {space }}$ approaches saturation, its values jump quickly for several bands, during which period the linearity of the detector is largely lost. This phenomenon is referred to as detector presaturation. The loss of linearity means that the calibrated gain coefficient $m$ measured at ICT temperature cannot represent the gain at EV samples, which essentially invalidate the IR calibration based on Eqs. (1) and (2). The impact of detector presaturation to gain calculation can also be observed in Fig. 6 from the scattered gain coefficients right before and after the detector saturation period.

The detector saturation/presaturation FPM temperatures are band dependent with small inband detector dependencies. The temperature thresholds at which saturation and presaturation occur are assessed at detector level with their band-median values listed in Table 3. The values are derived from a 7-day period centering at August 30, 2019, one of the hottest periods in a year with a peak FPM temperature of $107.8 \mathrm{~K}$. If the saturation temperature and presaturation temperature are very close for a band, it can be interpreted as the nonexistence of noticeable presaturation phenomenon for this band. Among these FPM temperature thresholds, the one for SL saturation can be conveniently referenced to determine the L1b data availability. From the calibration point of view, the ICT look presaturation temperature, the lowest among all, is a more precise cutoff threshold where the calibrated L1b becomes severely degraded. By different criteria, there are various other approaches developed by different teams to determine the FPM temperature thresholds. On April 8, 2019, the GPA was revised to add the FPM temperatures into every L1b file. L1b users then have the flexibility to customize the FPM temperature thresholds based on their specific applications.

\subsection{Noise Characterization}

Noise equivalent differential temperature (NEdT) is a key parameter for characterizing IR detector noise. ABI NEdT is calculated by the GPA for every ICT look, and the results are output in 
Wang et al.: On-orbit calibration and characterization of GOES-17 ABI IR bands...

Table 3 Detector saturation temperatures at gain set III (band median or $50 \%$ detectors, units: K).

\begin{tabular}{lcccc}
\hline \hline Band & ICT look presaturate & SL presaturate & ICT look saturate & SL saturate \\
\hline 8 & 94.2 & 95.3 & 94.6 & 96.1 \\
9 & 94.1 & 95.7 & 94.6 & 96.6 \\
10 & 93.2 & 93.9 & 93.8 & 94.8 \\
11 & 99.3 & 100.2 & 100.2 & 101.1 \\
12 & 91.7 & 92.1 & 92.3 & 92.5 \\
13 & 105.6 & 105.9 & 106.5 & 107.2 \\
14 & $\mathrm{~N} / \mathrm{A}$ & $\mathrm{N} / \mathrm{A}$ & $\mathrm{N} / \mathrm{A}$ & $\mathrm{N} / \mathrm{A}$ \\
15 & 95.6 & 96.1 & 98.2 & 98.7 \\
16 & 93.3 & 93.5 & 97.2 & 97.6 \\
\hline \hline
\end{tabular}

the INST-CAL files. The calculation follows the generic NEdT algorithm, which calculates the noise equivalent differential radiance (NEdN) first as the product of the detector gain coefficient $m$ and the standard deviation of the $x_{\mathrm{ICT}}$ and then converts the NEdN to NEdT by dividing the derivative of Planck's equation $\partial L / \partial T$. The fluctuation of GOES-17 ABI ICT temperature, ranging from 302 to $\sim 325 \mathrm{~K}$ daily, complicates the noise characterization since NEdT is scene temperature dependent. Therefore, the values saved in INST-CAL need to be scaled to $300 \mathrm{~K}$, at which temperature the NEdT specification is defined, ${ }^{26}$ to be compared with the specification.

$$
\mathrm{NEdT}_{300 \mathrm{~K}}=\frac{\mathrm{NEdT}_{T_{\mathrm{ICT}}} \times \partial L / \partial T_{T_{\mathrm{ICT}}}}{\partial L / \partial T_{300 \mathrm{~K}}}
$$

This correction does not consider the change of NEdN itself with the scene temperature, which is estimated to be a secondary effect.

The relationship between the NEdT and FPM temperature is shown in Fig. 13(a) for a sample date of August 28, 2019, at a peak FPM temperature of $~ 106 \mathrm{~K}$. As expected, the NEdT generally increases with the FPM temperatures. The drop of NEdT at higher FPM temperature is caused by the invalid gain calculation at detector saturation/presaturation, so it does not reflect the actual noise level corresponding to the FPM temperature, which is not necessarily known since the calibration already fails. At the same FPM temperature of $81 \mathrm{~K}$, when the detector is operated at gain set III, the NEdT is significantly larger than the NEdT at gain set I. This reflects an intrinsic characteristic of IR detectors that their noise level will be larger at lower detector gain. Larger NEdT is a compromise made when implementing low detector gain to alleviate detector saturation for extended data availability.

The NEdT performance of GOES-17 ABI is given in Table 4. The NEdT specifications in the table are the values defined prelaunch shared by GOES-16. The requirement waiver issued to GOES-17 after the discovery of the LHP anomaly is not taken into account. At the gain set I stage, band-averaged NEdT meets the specification except for bands 12 and 16. At gain set III, there are three scenarios.

1. The ICT look of bands 8 to 10 saturates before their NEdT exceeds the specification, so the NEdT of valid data is always below the specification.

2. The NEdT of bands 12, 15, and 16 always exceeds the specification.

3. The NEdT of bands 11,13 , and 14 is below the specification at $81 \mathrm{~K}$ and exceeds the specification at higher FPM temperatures. The FPM temperatures where NEdT exceed the specification are derived. 
Wang et al.: On-orbit calibration and characterization of GOES-17 ABI IR bands...

(a)
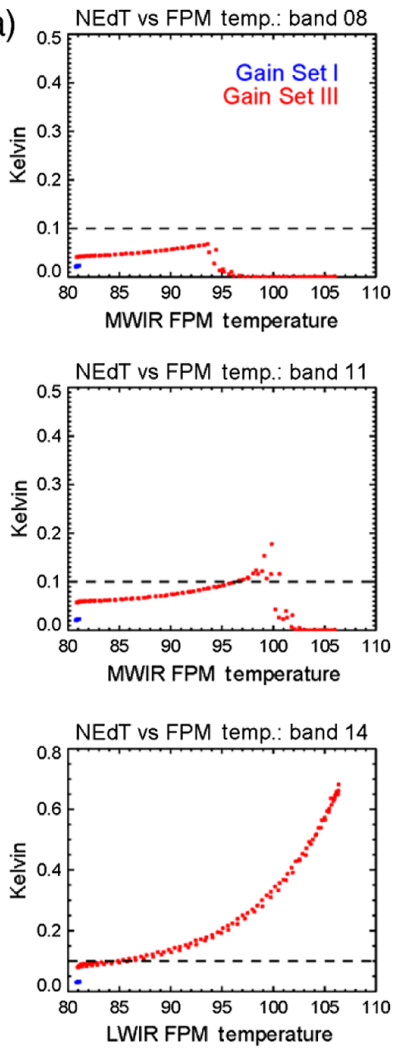

(b)
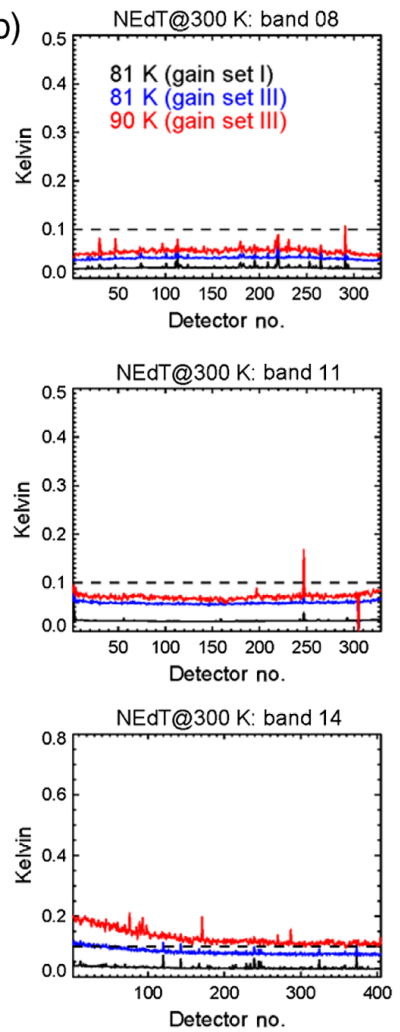
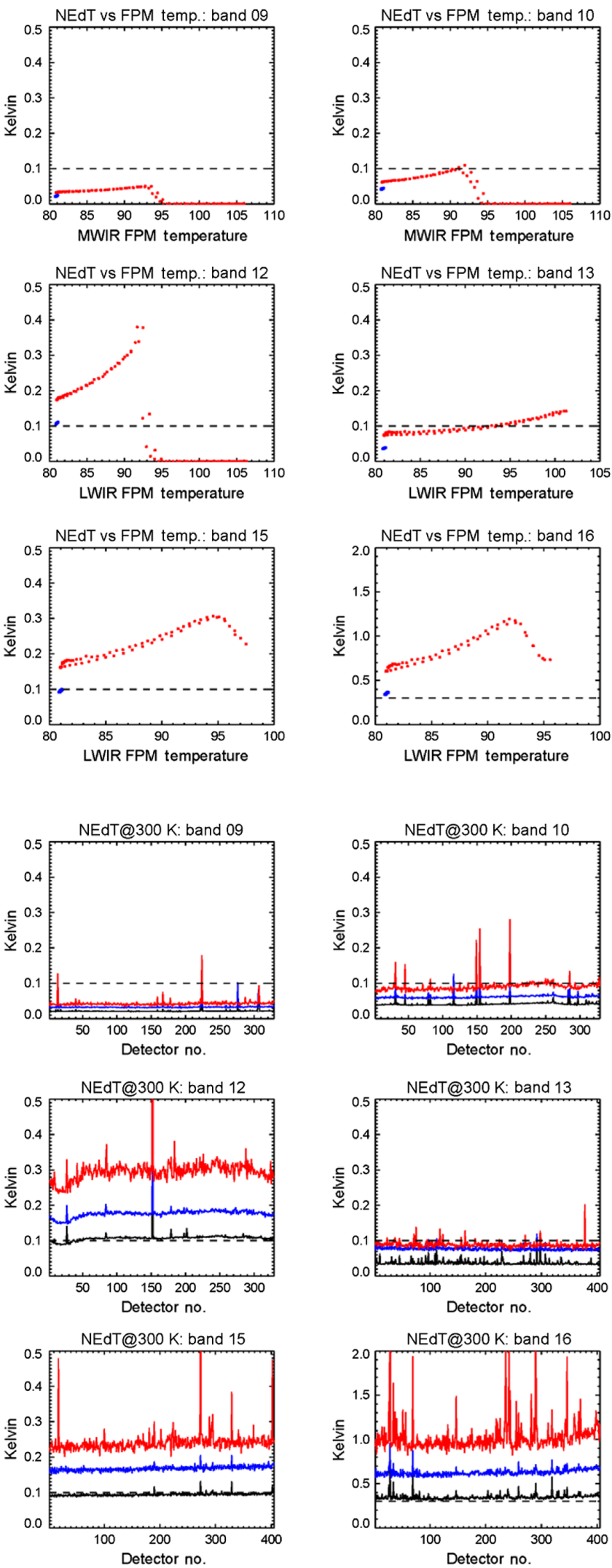

Fig. 13 The (a) relationship between the FPM temperature and the band-averaged NEdT and (b) the detector dependency of NEdT for August 28, 2019. 
Table 4 The mean (and standard deviation) of ABI IR bands NEdT at various FPM temperatures (units: K).

\begin{tabular}{|c|c|c|c|c|c|}
\hline Band & Original Spec. & $81 \mathrm{~K}$ (gain set I) & $81 \mathrm{~K}$ (gain set III) & $\begin{array}{l}\text { FPM temperature } \\
\text { when NEdT } \\
\text { exceeding spec. }\end{array}$ & GOES-16 $(60 \mathrm{~K})$ \\
\hline 8 & 0.1 & $0.022(0.004)$ & $0.041(0.004)$ & $\mathrm{N} / \mathrm{A}$ & $0.013(0.0006)$ \\
\hline 9 & 0.1 & $0.022(0.005)$ & $0.033(0.006)$ & $\mathrm{N} / \mathrm{A}$ & $0.015(0.0003)$ \\
\hline 10 & 0.1 & $0.042(0.011)$ & $0.063(0.009)$ & $\mathrm{N} / \mathrm{A}$ & $0.023(0.0005)$ \\
\hline 11 & 0.1 & $0.022(0.003)$ & $0.059(0.003)$ & 96.5 & $0.019(0.0003)$ \\
\hline 12 & 0.1 & $0.108(0.013)$ & $0.177(0.015)$ & Any & $0.018(0.0005)$ \\
\hline 13 & 0.1 & $0.037(0.007)$ & $0.077(0.006)$ & 92.7 & $0.028(0.0006)$ \\
\hline 14 & 0.1 & $0.031(0.006)$ & $0.083(0.011)$ & 84.8 & $0.019(0.0002)$ \\
\hline 15 & 0.1 & $0.094(0.004)$ & $0.167(0.007)$ & Any (gain set III) & $0.022(0.0005)$ \\
\hline 16 & 0.3 & $0.349(0.040)$ & $0.625(0.040)$ & Any & $0.034(0.0078)$ \\
\hline
\end{tabular}

In contrast, the NEdT of GOES-16 IR bands, operated at $60 \mathrm{~K}$, are all within the specifications and lower than their GOES-17 counterparts, as shown in Table 4. The within-band detector uniformity is also noticeably better.

The detector dependency of the NEdT has also been characterized. Overall, the NEdT of inband detectors of IR bands are comparable to each other. There are out-of-family detectors in all bands with NEdT that is significantly larger than other in-band detectors. Since NEdT performance is an intrinsic characteristics of a detector, this anomalous behavior cannot be improved by calibration alone. One solution used to reduce the NEdT of these out-of-family detectors is to identify them and take advantage of the detector redundancy provided by the ABI instrument. For ABI, each IR detector has six redundant detector elements manufactured side-by-side within a row, with only one enabled for operation at any given time. The set of the selected detector elements is called the best detector select (BDS) map, which was determined during the prelaunch and PLT stages, based on a set of criteria not limited to NEdT. For GOES-17 ABI, the BDS map determined could potentially still be further optimized due to the dramatic changes made to the detector gain setting. It is then possible, with this redundancy, to update the BDS map of these out-of-family detectors to yield better performance including NEdT reduction. Since launch, there have been multiple BDS map updates to the noisy or saturated detectors identified, and these updates did reduce the NEdT of these detectors as expected. However, out-of-family detectors still exist, as shown in Fig. 13(b) for August 28, 2019. This is either because the current detector element has the best performance already or the magnitudes of the noise of the detector change over time, so a then in-family detector becomes an out-of-family one. The detector elements currently in use are already considered the best available, so the noise performance is not expected to be further improved by BDS map update.

\subsection{Dynamic Range}

Operated at higher FPM temperature of $81 \mathrm{~K}$ and lower detector gain, the dynamic ranges of GOES-17 IR bands are mostly better than the Spec. and their GOES-16 counterparts during the gain set I stage except for band 13. The estimated detector saturation temperatures used for the full maturity review of L1b product are given in Table 5, derived from the data of January 7 , 2020. Their detector dependency is shown in Fig. 14. Similar to their noise performance, some detectors exhibit out-of-family behaviors with significantly lower saturation temperatures. $\mathrm{L} 1 \mathrm{~b}$ image saturation will always occur first for these detectors at lower scene temperatures. The saturation temperatures are even higher when gain set III is just turned on or about to be turned off when the FPM temperature is still around $81 \mathrm{~K}$, attributing to the lower gain and suppressed 
Wang et al.: On-orbit calibration and characterization of GOES-17 ABI IR bands...

Table 5 The saturation temperatures of $A B I I R$ bands (band minimum, units: $K$ ).

\begin{tabular}{lcccc}
\hline \hline Band & Spec. & 81 K (gain set I) & 81 K (gain set III) & GOES-16 $(60 \mathrm{~K})$ \\
\hline 8 & 300 & 354 & 438 & 335 \\
9 & 300 & 361 & 424 & 336 \\
10 & 320 & 391 & 515 & 368 \\
11 & 330 & 356 & 565 & 394 \\
12 & 330 & 580 & 847 & 346 \\
13 & 400 & 369 & 693 & 424 \\
14 & 330 & 397 & 738 & 378 \\
15 & 330 & 708 & 1170 & 402 \\
16 & 305 & 1793 & 3651 & 406 \\
\hline \hline
\end{tabular}
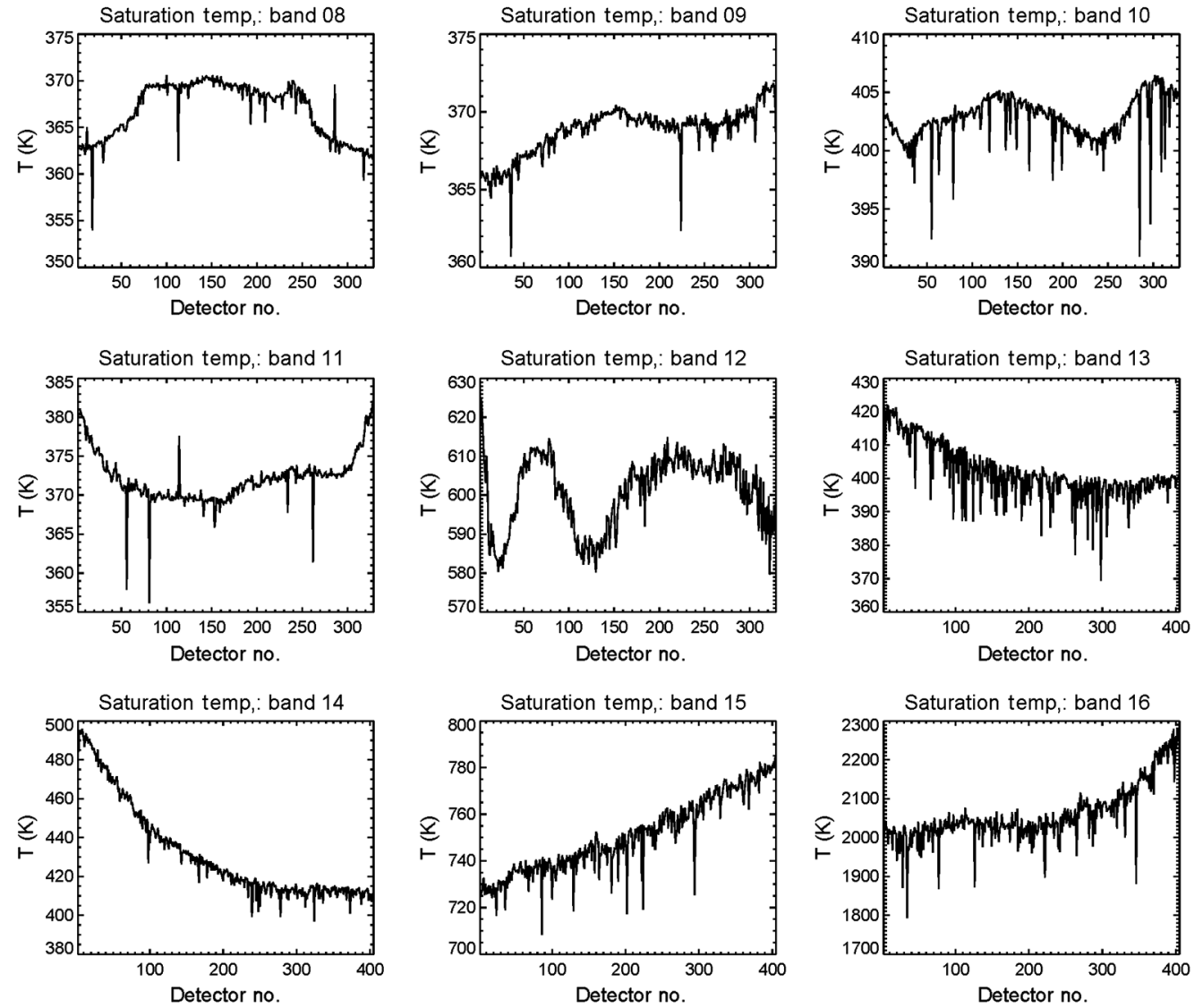

Fig. 14 The detector dependency of the saturation temperatures of GOES-17 IR bands when operated at gain set I and held stable at $81 \mathrm{~K}$.

SL counts shown in Fig. 4. The dynamic range will shrink when the FPM temperature continues to rise until the SL saturation occurs, at which point the dynamic range of the detector becomes zero.

The assessment of the dynamic range is associated with the study of the detector saturation/ presaturation in the above sections. Calculating the saturation temperatures as a function of FPM temperatures and using the results to fully characterize the dynamic range have been considered. 
There is a major weakness with this approach: the linearity at higher FPM temperature cannot be conserved as studied earlier, so the estimation of the saturation temperatures, which assumes a linear relationship between the counts and radiance, will not be accurate. It is unclear that such complex metrics are practically meaningful to the L1b users. Therefore, only the dynamic range in gain set I is assessed. For gain set III, it is recommended for the L1b users to directly use FPM temperature as the metric to estimate the image quality.

\subsection{IR Performance Overview}

Since the performance of IR GOES-17 ABI strictly correlates to the FPM temperature, and the FPM temperatures of GOES-17 ABI are seasonally and diurnally oscillating, the performance of its IR bands is temporally dynamic. It cannot be conveniently represented in traditional ways using the temporal averages of several parameters such as band-average NEdT and dynamic range. One solution for summarizing the performance is to determine the performance zones at any given time, following the definition in Fig. 11, and compiling them. A recent update of this IR performance summary, covering a period from August 1, 2018, to October 31, 2019, is shown in Fig. 15. The input data to the charts are all actual on-orbit calibration data, including the $x_{\text {space }}, x_{\mathrm{ICT}}$, and NEdT from INST-CAL files and ICT temperatures from the INSTCAL-ENG files. In these charts, the $x$ axis labels the time in a calendar day in UTC time and the $y$ axis labels the calendar date. Thus, the diurnal variation of the IR performance is presented along the $x$ direction, and the seasonal oscillation is presented along the $y$ direction. The performance of ABI IR bands is thus illustrated at any given time.

The shades of the green and gray color regions in Fig. 15 are the linear projection of the NEdT scaled to $300 \mathrm{~K}$ : darker colors represent larger NEdT. Blank spaces in these charts correspond to the time periods when the INST-CAL data were missing or invalid. For every band, there is a wider gap near the bottom that corresponds the satellite drift from its checkout location to the GOES-West operational location from October 24, 2018, to November 13, 2018, during
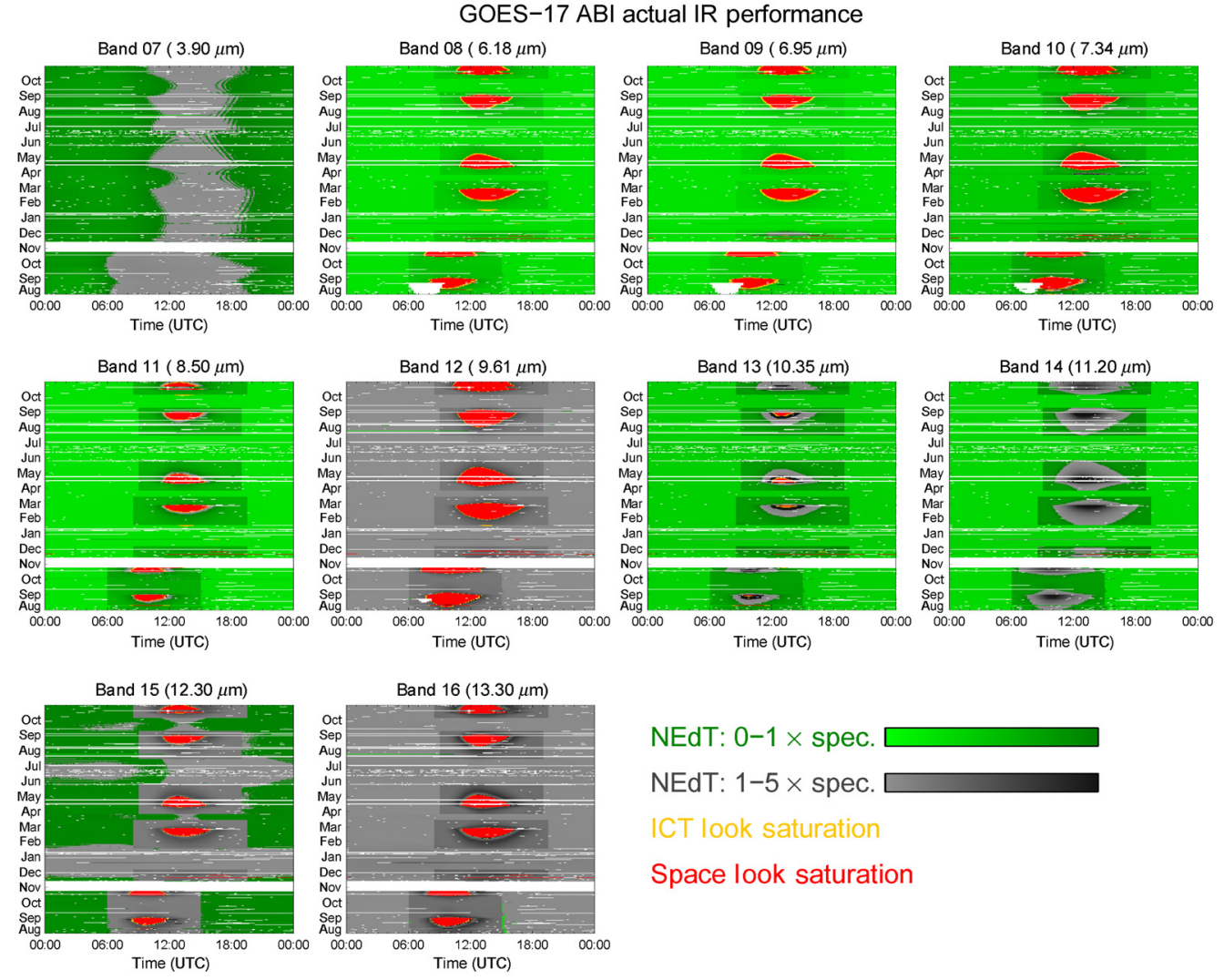

Fig. 15 GOES-17 ABI IR band performance overview from August 1, 2018, to October 31, 2019. 
which period the science data were not collected by the instrument. The checkout position was at $89.5 \mathrm{deg}$ W, nearly $45 \mathrm{deg}$ to the east of operational position. Therefore, the peak FPM temperatures occur $\sim 3 \mathrm{~h}$, in UTC time, before postdrift. For every band, there are several rectangular regions at darker shades than neighboring regions. This drastic change in shade is the result of NEdT change caused by gain set switch. Darker regions denote the gain set III region, and lighter regions denote the gain set I region. The regions have different widths because the gain switch occurred at 0830 UTC and 1930 UTC for summer and 0900 UTC and 1900 UTC for winter. These regions are segregated because the switch is enabled only for the days when their daily maximum FPM temperatures exceed $85 \mathrm{~K}$.

Critical information on GOES-17 ABI IR performance can be extracted from Fig. 15 visually. For example, it can be seen clearly from the colors that band 14 is the only IR band, other than band 7, that is immune from detector saturation. It is also seen that its noise performance is much worse than band 13, which complicates the user's choice of a better reference window band. On the other hand, many of the other miniature features in the charts, in particular for the time period before its orbit drift, are the results of miscellaneous instrument anomalies or instrument tests executed during the PLTs that changed the instrument behavior. Overall, these charts can serve as either a straightforward overview of IR performance for the L1b users, who do not necessarily have the comprehensive knowledge of calibration, or a record of calibration events for calibration analysts.

\section{Summary}

The GOES-17 ABI is experiencing an anomaly in its LHP subsystem that seriously deteriorates the cooling capability of the instrument. Through the optimization of instrument setting coordinated by the GOES-R Program, the heat load to the instrument is greatly reduced and the functionality of ABI IR bands is largely restored. Significant improvements have been made to improve the ABI L1b radiance product through calibration. These include several builds of software upgrade that includes the implementation of $\mathrm{pCal}$ to reduce calibration bias.

Still, the MWIR/LWIR bands are operated at elevated and varying FPM temperatures within a range from 81 to $108 \mathrm{~K}$, compared with the design value of $60 \mathrm{~K}$. Since the performance of IR detectors relies strongly on the operating temperatures, the GOES-17 ABI's IR performance cannot compete with the design values and GOES-16 ABI. In this paper, the relationship between the IR detector performance and the FPM temperature is extensively studied, and the evaluation of key IR performance parameters, such as calibration bias, noise, and dynamic range, is made. For $\sim 70 \%$ of the time, the IR detectors are running at the gain set I stage, which corresponds to a relatively stable FPM temperature of $81 \mathrm{~K}$. During this period, major design Specs. related to IR L1b quality are still met. Larger NEdT of bands 12, 15, 16 and lower dynamic range for band 13 could impact the users of these bands. For the remaining $30 \%$ of the time when the IR bands are utilizing the gain set III, which corresponds to dynamic temperatures, the detectors performance is worse than at gain set I. The original requirements of all of the above parameters will be exceeded at certain high temperatures for all of these bands. For the L1b users, the selection of good data will be primarily based on the FPM temperatures saved in the L1b data files, while the results provided in the paper are expected to help the users to understand the quality of the selected data. The health and performance of the instrument will be continuously monitored and tracked by the CWG to support the production of high-quality ABI L1b products to meet users' needs.

\section{Acknowledgments}

The authors would like to thank current and former members of the GOES-R CWG and other teams collaboratively working on the GOES-R calibration/validation ( $\mathrm{Cal} / \mathrm{Val})$, in particular the GOES-R Algorithm Working Group and the PRO team, for their efforts and contributions to the prelaunch and on-orbit Cal/Val of GOES-R ABI instruments. Abundant resources on ABI and its $\mathrm{Cal} /$ Val are available online. Other than those referenced, for example, similar GOES-16/GOES17 intersensor comparison results, which also include thumbnails of the imagery and the data quality flag values, are generated at the CIMSS. ${ }^{27}$ This study was supported through the 
GOES-R Program and the GOES-R Flight project. The views and opinions in this paper are solely those of the authors and do not constitute a statement of policy, decision, or position on behalf of NOAA or the U.S. Government. Distribution Statement A: Approved for public release. Distribution is unlimited. This material is based upon work supported by the National Oceanic and Atmospheric Administration under Air Force Contract No. FA8702-15-D-0001. Any opinions, findings, conclusions or recommendations expressed in this material are those of the author(s) and do not necessarily reflect the views of the National Oceanic and Atmospheric Administration. Delivered to the U.S. Government with Unlimited Rights, as defined in DFARS Part 252.227-7013 or 7014 (Feb 2014). Notwithstanding any copyright notice, U.S. Government rights in this work are defined by DFARS 252.227-7013 or DFARS 252.227-7014 as detailed above. Use of this work other than as specifically authorized by the U.S. Government may violate any copyrights that exist in this work.

\section{References}

1. GOES-R Series Program, "GOES-R series product definition and users' guide," 2018, https://www.goes-r.gov/products/docs.

2. T. J. Schmit et al., "Introducing the next-generation Advanced Baseline Imager on GOES-R," Bull. Am. Meteorol. Soc. 86, 1079-1096 (2005).

3. T. J. Schmit et al., "A closer look at the ABI on the GOES-R series," Bull. Am. Meteorol. Soc. 98, 681-698 (2017).

4. S. Kalluri et al., "From photons to pixels: processing data from the Advanced Baseline Imager," Remote Sens. 10(2), 177 (2018).

5. T. J. Schmit et al., "Applications of the 16 spectral bands on the Advanced Baseline Imager (ABI)," J. Oper. Meteorol. 6(4), 33-46 (2018).

6. GOES-R Series Program, "GOES-R series data book," 2019, https://www.goes-r.gov/ downloads/resources/documents.

7. X. Xiong et al., "Terra and Aqua MODIS thermal emissive bands on-orbit calibration and performance," IEEE Trans. Geosci. Remote Sens. 53(10), 5709-5721 (2015).

8. C. Cao et al., "Suomi NPP VIIRS sensor data record verification, validation, and long-term performance monitoring," J. Geophys. Res. Atmos. 118(20), 11,664-11,678 (2013).

9. X. Xiong et al., "VIIRS on-orbit calibration methodology and performance," J. Geophys. Res. Atmos. 119(9), 5605-5078 (2014).

10. W. Lebair et al., "Post launch calibration and testing of the Advanced Baseline Imager on the GOES-R satellite," Proc. SPIE 9881, 98810K (2016).

11. J. V. Naarden and D. Lindsey, "Saving GOES-17,” 2019, https://aerospaceamerica.aiaa.org/ departments/saving-goes-17.

12. J. McCorkel et al., "GOES-17 Advanced Baseline Imager performance recovery summary," in IEEE Int. Geosci. and Remote Sens. Symp., Yokohama, Japan (2019).

13. M. R. Black et al., "Mitigation of secondary radiometric calibration artifacts in \{GOES-17 advanced baseline imager\} data," in Joint Satellite Conf. , Boston (2019).

14. J. P. Fulbright et al., "Calibration/validation strategy for GOES-R L1b data products," Proc. SPIE 10000, 100000T (2016).

15. F. Yu et al., "Early radiometric calibration performances of GOES-16 Advanced Baseline Imager," Proc. SPIE 10402, 104020S (2017).

16. H. Qian et al., "Detection and characterization of striping in GOES-16 ABI VNIR/IR bands," Proc. SPIE 10764, 107641N (2018).

17. Z. Wang et al., "Radiometric quality assessment of GOES-16 ABI L1b images," Proc. SPIE 10764, 107641T (2018).

18. F. Yu et al., "Radiometric calibration performance of GOES-17 Advanced Baseline Imager (ABI)," Proc. SPIE 11127, 111271C (2019).

19. GOES-R Series Program, https://www.goes-r.gov/users/GOES-17-ABI-Performance.html (2020).

20. A. I. D'Souza et al., "Detectors and focal plane modules for weather satellites," Proc. SPIE 9881, 988115 (2016). 
21. Z. Wang et al., "Monitoring and assessment of the temperature fluctuation of Aqua MODIS cold focal plane assembly," Proc. SPIE 8510, 85100K (2012).

22. Z. Wang et al., "Update on the status of Aqua MODIS cold focal plane assembly temperature fluctuation," Proc. SPIE 10402, 104021R (2017).

23. H. Yoo, F. Yu, and X. Wu, "Evaluation of radiometric calibration consistency of GOES-17 ABI using GEO-GEO collocations," in AGU Fall Meeting (2018).

24. GOES-R CWG, "GOES-17 ABI-GOES-16 ABI Tb difference," https://www.star.nesdis .noaa.gov/GOESCal/G17_ABI_GEO_GEO_IR_daily.php (2020).

25. L. Wang et al., "Inter-comparison of NPP/CrIS radiances with VIIRS, AIRS, and IASI: a post-launch calibration assessment," Proc. SPIE 8528, 85280J (2012).

26. GOES-R Series Program, "GOES-R mission requirements document," https://www.goes-r .gov/syseng/docs/MRD.pdf (2020).

27. UW-Madison/CIMSS, "GOES-R series activities," http://cimss.ssec.wisc.edu/goes-r/abi-/ band_statistics_imagery.html (2020).

28. UW-Madison/Space Science and Engineering Center, https://www.ssec.wisc.edu/data/ (2020).

29. NOAA/Office of Satellite and Product Operations (OSPO), "GOES schedules and scan sectors," https://www.ospo.noaa.gov/Operations/GOES/schedules.html (2020).

Zhipeng (Ben) Wang is an associate research scientist at the Earth System Science Interdisciplinary Center, University of Maryland, College Park. As a member of NOAA's GOES-R CWG, his main responsibility was to monitor the performance of the $\mathrm{ABI}$ instruments to support their on-orbit calibration and characterization and to resolve miscellaneous calibration anomalies.

Biographies of the other authors are not available. 